\title{
Projection effects in galaxy cluster samples: insights from X-ray redshifts
}

\author{
M. E. Ramos-Ceja ${ }^{1}$, F. Pacaud ${ }^{1}$, T. H. Reiprich ${ }^{1}$, K. Migkas ${ }^{1}$, L. Lovisari ${ }^{2}$, and G. Schellenberger ${ }^{2}$ \\ 1 Argelander-Institut für Astronomie, Universität Bonn, Auf dem Hügel 71, 53121 Bonn, Germany \\ e-mail: miriam@astro.uni-bonn.de \\ 2 Center for Astrophysics, Harvard \& Smithsonian, 60 Garden Street, Cambridge, MA 02138, USA
}

Received 23 January 2019 / Accepted 19 April 2019

\begin{abstract}
Presently, the largest sample of galaxy clusters selected in X-rays comes from the ROSAT All-Sky Survey (RASS). Although there have been many interesting clusters discovered with the RASS data, the broad point spread function of the ROSAT satellite limits the attainable amount of spatial information for the detected objects. This leads to the discovery of new cluster features when a re-observation is performed with higher-resolution X-ray satellites. Here we present the results from XMM-Newton observations of three clusters: RXC J2306.6-1319, ZwCl 1665, and RXC J0034.6-0208, for which the observations reveal a double or triple system of extended components. These clusters belong to the extremely expanded HIghest X-ray FLUx Galaxy Cluster Sample (eeHIFLUGCS), which is a flux-limited cluster sample $\left(f_{\mathrm{X}, 500} \geq 5 \times 10^{-12} \mathrm{erg} \mathrm{s}^{-1} \mathrm{~cm}^{-2}\right.$ in the $0.1-2.4 \mathrm{keV}$ energy band). For each structure in each cluster, we determine the redshift with the X-ray spectrum and find that the components are not part of the same cluster. This is confirmed by an optical spectroscopic analysis of the galaxy members. Therefore, the total number of clusters is actually seven, rather than three. We derive global cluster properties of each extended component. We compare the measured properties to lower-redshift group samples, and find a good agreement. Our flux measurements reveal that only one component of the $\mathrm{ZwCl} 1665$ cluster has a flux above the eeHIFLUGCS limit, while the other clusters will no longer be part of the sample. These examples demonstrate that cluster-cluster projections can bias X-ray cluster catalogues and that with high-resolution X-ray follow-up this bias can be corrected.
\end{abstract}

Key words. X-rays: galaxies: clusters - large-scale structure of Universe - galaxies: clusters: intracluster medium

\section{Introduction}

Systematic searches for galaxy clusters have traditionally been conducted at optical wavelengths (e.g. Abell 1958), with the main selection criterion being the statistical excess of cluster galaxies with respect to the background along the line of sight. While some optical catalogues are still the largest compilations of galaxy clusters (e.g. Wen et al. 2012), the inherent biases to the optical selection process, most importantly projection effects, lead to false detections that are difficult to correct for in statistical studies of cluster properties (e.g. Costanzi et al. 2019).

In the last two decades X-ray and sub-millimetre observations have provided some of the purest galaxy cluster catalogues (e.g. Böhringer et al. 2000, 2001; Planck Collaboration XXVII 2016). Although these samples tend to detect a particular type of cluster (see Giodini et al. 2013, for a description of several biases), they overcome most of the optical biases. Specifically, $\mathrm{X}$-rays provide powerful means of selecting galaxy clusters and characterising their properties. This is possible because the $\mathrm{X}$-ray flux is proportional to the square of the electron density of the intra-cluster medium (ICM), and therefore it is less prone to line-of-sight structure projection. Furthermore, the gas only gets hot enough to emit $\mathrm{X}$-rays if it sits in a deep gravitational potential. Last but not least, in X-rays, clusters are identified as single objects, that is, as huge hot plasma clouds, and not just as the sum of individual galaxies. X-ray observations of galaxy clusters therefore allow for the compilation of cluster samples that are almost unaffected by projection effects and have very few false detections. Finally, the analysis of the X-ray emission spectrum of the ICM also allows the determination of various cluster properties such as for example the gas mass, temperature, metal abundance, and the redshift of the cluster. The ICM is a plasma that can generally be assumed to be optically thin in collisional equilibrium, and whose spectrum is described by a bremsstrahlung spectrum together with emission lines of highly ionised metals (e.g. Sarazin 1986; Peterson \& Fabian 2006; Böhringer \& Werner 2010).

Cluster redshifts and total masses are crucial measurements for cosmological studies based on galaxy clusters. The cluster redshift information is usually obtained from optical spectroscopic follow-up observations of the cluster member galaxies, however this process is limited to one or a few cluster member galaxies and can require long observational campaigns for large cluster samples. While these spectroscopic measurements give very precise cluster redshifts $(\Delta z /(1+z)<0.01)$, the direct use of $\mathrm{X}$-ray data also yields complementary redshift estimations (e.g. Yu et al. 2011; Lloyd-Davies et al. 2011; Liu et al. 2018).

X-ray cluster catalogues based on the ROSAT All-Sky Survey (RASS, Truemper 1992, 1993), such as the Northern ROSAT All-Sky Survey (NORAS, Böhringer et al. 2000), the Bright Cluster Sample (BCS, Ebeling et al. 1998), and the ROSATESO Flux Limited X-ray Galaxy Cluster Survey (REFLEX, Böhringer et al. 2001) typically consist of a few hundred clusters. However, due to the limited RASS spatial resolution (of order $1 \mathrm{arcmin}$ ) and the limited galaxy redshift follow-up, a fraction of the systems identified as single clusters may indeed be double clusters or even two unrelated clusters with a small projected separation. The XMM-Newton and Chandra 
Table 1. Cluster properties extracted from the MCXC catalogue.

\begin{tabular}{lcccccc}
\hline \hline Cluster & $\begin{array}{c}\text { RA } \\
(\mathrm{J} 2000)\end{array}$ & $\begin{array}{c}\text { Dec } \\
(\mathrm{J} 2000)\end{array}$ & $z$ & $\begin{array}{c}r_{500} \\
(\mathrm{kpc})\end{array}$ & $\begin{array}{c}L_{500} \\
\left(10^{43} \mathrm{erg} \mathrm{s}^{-1}\right)\end{array}$ & $\begin{array}{c}\text { Parent } \\
\text { catalogue }\end{array}$ \\
\hline RXC J2306.6-1319 & 346.650 & -13.320 & 0.066 & 772 & 5.7 & REFLEX \\
ZwCl 1665 & 125.798 & +4.356 & 0.029 & 630 & 1.9 & BCS \\
RXC J0034.6-0208 & 8.650 & -2.140 & 0.081 & 897 & 12.5 & REFLEX \\
\hline
\end{tabular}

Notes. The luminosity is given in the $0.1-2.4 \mathrm{keV}$ energy band.

observatories have larger effective areas and higher angular resolution than ROSAT, allowing for a deeper analysis of the ICM, helping us to identify such double cluster systems. For example, upon close inspection with XMM-Newton, the cluster Abell 1644 turned out to be a merging double cluster (Reiprich et al. 2004). Moreover, the substructure selection has been an important issue when using complete galaxy cluster samples for cosmological analysis; for example, Schellenberger \& Reiprich (2017) developed and employed an objective, automated substructure-removal procedure that works well with high-quality data.

In the near future, the extended ROentgen Survey with an Imaging Telescope Array (eROSITA, Predehl et al. 2010; Merloni et al. 2012) is expected to detect $\sim 10^{5}$ clusters (Pillepich et al. 2012, 2018; Clerc et al. 2018). The cosmological studies that will be carried out with these detected clusters will require the knowledge of the cluster redshift. For eROSITA, this will be approached in different ways: lower-precision photometric redshifts are readily available through large-area multicolour surveys like DES (Dark Energy Survey Collaboration 2016), ATLAS (Shanks et al. 2015), and PanSTARRS (Chambers 2016). Special projects to acquire higher-precision spectroscopic redshifts are underway (e.g. 4MOST and SPIDERS, de Jong et al. 2014; Clerc et al. 2016; Zhang et al. 2017). Additionally, low-precision $(\Delta z /(1+z)<0.1) \mathrm{X}$-ray redshifts will be directly available from the eROSITA survey data for a subsample of clusters (Borm et al. 2014). It is also expected that high-resolution data from $X M M$ Newton, Chandra, and XRISM (Tashiro et al. 2018) will be available for a subsample of the order of $10^{3}$ clusters.

In this paper, we report on the XMM-Newton observations of three clusters detected in the RASS, for which the XMM-Newton data reveal a double or even triple X-ray morphology. We use X-ray and optical spectroscopy information and find that the clusters detected in the RASS data are not only not single clusters but are even simply projection effects and that the double $\mathrm{X}$-ray morphologies are indeed separated clusters at different redshifts. Throughout this paper, we assume a flat $\Lambda \mathrm{CDM}$ cosmology with $\Omega_{\mathrm{m}}=0.3$ and $H_{0}=70 \mathrm{~km} \mathrm{~s}^{-1} \mathrm{Mpc}^{-1}$.

\section{Selected clusters}

The extremely expanded HIghest X-ray FLUx Galaxy Cluster Sample (eeHIFLUGCS, Reiprich 2017) comprises all clusters with $f_{\mathrm{X}, 500} \geq 5 \times 10^{-12} \mathrm{erg} \mathrm{s}^{-1} \mathrm{~cm}^{-2}$ in the $0.1-2.4 \mathrm{keV}$ energy band (almost 400 clusters). This selection takes into account only RASS-based catalogues, whose homogenized fluxes are calculated from the luminosities determined in the Meta-Catalog of X-Ray Detected Clusters of Galaxies (MCXC, Piffaretti et al. 2011). As of October 2018, good-quality data in XMM-Newton and/or Chandra is available for $\sim 87 \%$ of the sample ( $\sim 330$ clusters). Using images in the $0.5-2 \mathrm{keV}$ energy band we perform a visual inspection of the good XMM-Newton observations ( 240 clusters) and identify that approximately 15 clusters show two or more clear extended structures, that is, objects with diameters larger than 2 arcmin. These objects are either merging systems or uncorrelated objects along the line of sight. In this work, we present three clusters that fall into the latter category. In this work we present the X-ray spectral analysis that allows us to determine that the extended objects present in these observations have different redshifts from the one reported in MCXC and redshifts that are distinct among themselves.

In the following, we present the previously known X-ray information for the three clusters. Table 1 shows a summary of the most important known features (from Piffaretti et al. 2011).

\section{1. $R X C J 2306.6-1319$}

According to the MCXC, the cluster RXC J2306.6-1319 (MCXC J2306.5-1319) has been catalogued in REFLEX at $z=$ 0.066 , with $r_{500}=0.772 \mathrm{Mpc}^{1}(\sim 10.17 \mathrm{arcmin})$ and a luminosity $L_{500}=5.74 \times 10^{43} \mathrm{erg} \mathrm{s}^{-1}$ in the $0.1-2.4 \mathrm{keV}$ rest-frame energy band.

RXC J2306.6-1319 is located $\sim 9.6$ arcmin from another MCXC cluster, RXC J2306.8-1324 (MCXC J2306.8-1324). This cluster has been identified in the SGP (A catalogue of clusters of galaxies in a region of 1 steradian around the South Galactic Pole, Cruddace et al. 2002) at $z=0.066$, with $r_{500}=$ $0.710 \mathrm{Mpc}(\sim 9.36 \mathrm{arcmin})$, and $L_{500}=3.81 \times 10^{43} \mathrm{erg} \mathrm{s}^{-1}$ in the $0.1-2.4 \mathrm{keV}$ energy band. Böhringer et al. (2004) have a note on these clusters: RXC J2306.6-1319 is one out of nine REFLEX clusters not listed in SGP, while RXC J2306.8-1324 is one out of six clusters listed in the SGP with a flux above the REFLEX flux limit, but in REFLEX it has a flux lower than the REFLEX flux limit.

\section{2. $\mathrm{ZwCl} 1665$}

The MCXC cluster ZwCl1665 (MCXC J0823.1+0421) has been catalogued in the BCS at $z=0.029$, with $r_{500}=$ $0.630 \mathrm{Mpc}(\sim 17.88 \mathrm{arcmin})$ and $L_{500}=1.92 \times 10^{43} \mathrm{erg} \mathrm{s}^{-1}$ in the $0.1-2.4 \mathrm{keV}$ energy band.

\section{3. $R X C J 0034.6-0208$}

RXC J0034.6-0208 (MCXC J0034.6-0208) has been identified in the REFLEX catalogue at $z=0.081$, with $r_{500}=0.897 \mathrm{Mpc}$ $(\sim 9.77 \mathrm{arcmin})$ and $L_{500}=1.25 \times 10^{44} \mathrm{erg} \mathrm{s}^{-1}$ in the $0.1-2.4 \mathrm{keV}$ energy band. In the REFLEX catalogue there is an additional note about this cluster: "DL, two maxima/E-W", which we interpret as a double cluster displaying two X-ray maxima in the east-west direction. Moreover, RXC J0034.6-0208 has

\footnotetext{
${ }^{1} r_{500}$ is the radius within which the mean over-density of the galaxy cluster is 500 times the critical density at the cluster redshift.
} 
M. E. Ramos-Ceja et al.: Projection effects in galaxy cluster samples: insights from X-ray redshifts

Table 2. Observation ID, unfiltered time of pn, clean exposure time of each EPIC camera, IN/OUT ratio, and hydrogen column density value for the three observations used in this paper.

\begin{tabular}{lccccccc}
\hline \hline Cluster & Obs. ID & $\begin{array}{c}\text { Total } \\
(\mathrm{ks})\end{array}$ & $\begin{array}{c}\text { MOS1 } \\
(\mathrm{ks})\end{array}$ & $\begin{array}{c}\text { MOS2 } \\
(\mathrm{ks})\end{array}$ & $\begin{array}{c}\mathrm{pn} \\
(\mathrm{ks})\end{array}$ & $\begin{array}{c}\text { IN/OUT ratio } \\
\text { MOS1/MOS2/pn }\end{array}$ & $\begin{array}{c}n_{\mathrm{H}} \\
\left(10^{20} \mathrm{~cm}^{-2}\right)\end{array}$ \\
\hline RXC J2306.6-1319 & 0765030201 & 11.4 & 8.1 & 8.92 & 5.8 & $1.061 / 1.118 / 1.010$ & 3.0 \\
ZwCl 1665 & 0741580501 & 12.6 & 10.5 & 15.3 & 9.9 & $1.077 / 1.006 / 1.098$ & 2.5 \\
RXC J0034.6-0208 & 0720250401 & 11.2 & - & 7.3 & 11.0 & $-/ 1.001 / 0.961$ & 3.1 \\
\hline
\end{tabular}

been associated with one Planck cluster: PSZ2 G113.02-64.68 (Planck Collaboration XXVII 2016).

At a distance of $~ 9.6$ arcmin from RXC J0034.6-0208, the MCXC cluster RXC J0034.2-0204 (MCXC J0034.2-0204) is located. This cluster has also been identified in the SGP catalogue at $z=0.082$, with $r_{500}=0.893 \mathrm{Mpc}(\sim 9.62 \mathrm{arcmin})$ and $L_{500}=1.23 \times 10^{44} \mathrm{erg} \mathrm{s}^{-1}$ in the $0.1-2.4 \mathrm{keV}$ energy band. This cluster is also known as SH518 (Shectman 1985).

The case of RXC J0034.6-0208 is very similar to RXC J2306.6-1319. According to Böhringer et al. (2004), RXC J0034.6-0208 is one out of nine REFLEX clusters not listed in SGP.

\section{XMM-Newton observations and data analysis}

The three galaxy clusters described in Sect. 2 have been observed by XMM-Newton. RXC J0034.6-0208 has been observed four times with XMM-Newton (Obs. ID 0675470901, 0675472601, 0720250401, 0720252901), but three of them are entirely flared and cannot be used for a spectral analysis. Only Obs. ID 0720250401 (taken with the thin filter) has usable data, but only for the MOS2 and pn detectors. MOS1 presented some anomalies and did not work in the necessary conditions to collect data. The observations for RXC J2306.6-1319 and ZwCl 1665 were taken with the medium filter (see Table 2 for the Obs. ID), and all three observations were set to Full Frame mode. ZwCl 1665 has also been observed with Chandra ( 10 ks). We briefly discuss some results in Sect. 4.2.

\subsection{Data reduction}

We retrieved the observation data files (ODFs) from the $X M M$-Newton archive and reprocessed them with the XMMSAS v16.0.0 software. We used the tasks emchain and epchain to generate calibrated event lists from the ODFs.

We performed a filtering process to clean the data from periods of soft-proton flares. For this we created a light curve in the $0.3-10 \mathrm{keV}$ energy band with bins of $52 \mathrm{~s}$ for MOS and $26 \mathrm{~s}$ for pn, and selected only good events (by applying the expressions \#XMMEA_EM for MOS and (FLAG \& $0 \times 766 \mathrm{~b} 0000$ ) $==0$ for pn). Following Pratt \& Arnaud (2002), we fitted a Poisson distribution to the light curve, and applied a $\pm 3 \sigma$ thresholding. We rejected all time intervals exceeding this threshold and obtained the good time intervals (GTIs). Table 2 shows the clean exposure time of the observations.

Since a residual contribution from soft protons may still exist after the initial data filtering, we quantified this source of contamination for our data. For this, we determined the so-called IN/OUT ratio (De Luca \& Molendi 2004; Leccardi \& Molendi 2008), which is the ratio of the surface brightness inside the fieldof-view ( $\mathrm{FoV}$ ) to the one measured in the unexposed corners. The signal in the corners provides an estimate of the cosmic-ray induced instrumental background as focused X-ray photons or soft protons cannot pass through the filter support structure. On the other hand, performing the measurement at high energies ensures that the signal inside the $\mathrm{FoV}$ is also free from genuine $\mathrm{X}$-ray photons due to the low telescope effective area. Therefore, an excess of the IN/OUT ratio above unity indicates softproton contamination. De Luca \& Molendi (2004) have shown that IN/OUT ratios $<1.15$ indicate mostly clean observations while ratios $>1.3$ are a sign of severe soft-proton contamination. In practice, we relied on EPIC count-rates calculated in the energy band 6-12 keV for MOS and 5-7 and $10-14 \mathrm{keV}$ for PN, but excluded the central area 10 arcmin of the FoV to minimise contamination by the sky signal at medium energies. In addition, since the cosmic-ray induced instrumental background also shows spatial variations, we computed the IN/OUT ratio for similar regions, flags, and energies from our instrumental background template (Filter Wheel Closed, FWC, observations) and used it to renormalise our IN/OUT diagnostic. Experience shows that the criteria laid out by De Luca \& Molendi (2004) still apply with our modified version of the IN/OUT test. We list the measured IN/OUT ratio of each observation in Table 2. These point to a low contamination by residual soft-proton emission and therefore we neglect this possible contribution in the spectral fitting (see Sect. 3.2).

The Solar Wind Charge Exchange (SWCX, Carter \& Sembay 2008; Carter et al. 2011) may also be an important source of contamination in X-ray observations. We determine if our observations are affected by SWCX employing the method outlined in Carter \& Sembay (2008) and Carter et al. (2011). This procedure consists of comparing the count-rates of two light curves (with bin size of $1 \mathrm{ks}$ ): the continuum-band $(2.5-5 \mathrm{keV})$ and the line-band $(0.5-0.7 \mathrm{keV})$. A scatter plot between the two bands is produced and a linear model fit is computed. If a high reduced $\chi^{2}$ value $(\geq 1.2)$ is obtained from the fit, then the observation is considered to have a SWCX enhancement. Moreover, the $\chi^{2}$ values for each individual light curve in terms of the deviation from their respective mean light curve are calculated. The ratio between the $\chi^{2}$ value for the line band to the $\chi^{2}$ value for the continuum line is then calculated. Values $\geq 1.0$ of this ratio are expected in observations with high SWCX. None of the observations analysed in this work meet either of these two criteria, therefore we do not account for SWCX contamination in the spectral fitting analysis.

We have also checked for so-called "anomalous state" in the MOS detectors (Kuntz \& Snowden 2008). Using the $0.2-0.9 \mathrm{keV}$ energy band, we found that CCD no. 5 of the MOS2 detector in the $\mathrm{ZwCl} 1665$ observation has a strong enhancement of photons at such energies. This affected CCD is excluded from further processing.

We also obtain a list of out-of-time events, which are added to the instrumental background (see Sect. 3.2) and then subtracted from the data set.

For the detection of point sources, we used a two-stage procedure. First, we applied the source-detection method of 
Pacaud et al. (2006) in the 0.5-2 keV energy band. Briefly, we co-added images of the three EPIC detectors in this band, and the resulting image is filtered using the wavelet task MR_FILTER from the multiresolution package MR/1 (Starck et al. 1998). The source detection and catalogue production are performed by the SEXTRACTOR software (Bertin \& Arnouts 1996). In a second step, we examined by eye the co-added images in the soft and hard band, $0.5-2 \mathrm{keV}$ and $2-10 \mathrm{keV}$, respectively, to find possible undetected point sources. The corresponding regions of the final list of detected point sources were removed from the event lists in the subsequent analysis.

\subsection{X-ray spectral analysis}

We performed a spectral analysis of the clusters in the $0.4-10 \mathrm{keV}$ energy band. The fitting procedure was performed using XSPEC v12.9.1h. In the following, we describe the modelling of the background and of the source.

\subsubsection{Background modelling}

It is well known that the modelling of the background is crucial to obtain reliable measurements of the properties of the ICM. The total background in X-ray observations consists of two main components: the non-vignetted particle background (PB) and the cosmic X-ray background (CXB).

In XMM-Newton observations, the $\mathrm{PB}$ consists of a continuum component and fluorescence X-ray lines (see Snowden et al. 2008, for details). These lines affect the MOS and pn detectors differently: there are strong $\mathrm{Al}$ and $\mathrm{Si}$ lines in the $1.4-1.9 \mathrm{keV}$ energy band mainly affecting the MOS detectors, and other lines, for example, $\mathrm{Ni}, \mathrm{Cu}$, and $\mathrm{Zn}$, around the 7.4-9 keV energy band showing in the pn detector. Since FWC observations are dominated by the instrumental background; we use them to subtract the internal instrumental background from our observations. For this, FWC observations must be renormalized to match the level of PB present in the observations. Following the conclusions of Kuntz \& Snowden (2008), we renormalise the FWC data independently for each MOS CCD or PN quadrant using their individual corners. For the MOS central CCDs, we compute the normalisation based on the corners of the surrounding CCDs that best correlate with them $([2,3,6,7]$ for MOS1 and [3,4,6] for MOS2). Zhang et al. (2009) found that the 3-10 keV energy band gives good re-normalisation factors. We go a step further and narrow this energy band, avoiding the fluorescence X-ray lines present in the EPIC detectors. To calculate the FWC re-normalisation factors we compute the count-rate, in the source and FWC observations using photons out of the FoV for each CCD using the $2.5-5$ and $8-9 \mathrm{keV}$ energy bands for the MOS detectors and the $2.5-5 \mathrm{keV}$ energy band for the pn detectors. We include Gaussian line emission in the spectral fitting to account for residuals that exist after the PB subtraction.

The CXB is composed of a thermal-emission component from the Local Hot Bubble (LHB), a second thermal emission component from the Galactic halo, and an extra-galactic component representing the unresolved emission from active galactic nuclei (AGNs). Given that these distinct components have different spectral features, this background is more difficult to subtract from the observations. Instead, we model and fit the CXB components when doing the spectral analysis of our sources. We follow a similar spectral-fitting methodology to the one described in Snowden et al. (2008). The CXB is well described by a model that includes an absorbed $\sim 0.2 \mathrm{keV}$ thermal compo- nent representing the Galactic Halo emission (McCammon et al. 2002), an unabsorbed $\sim 0.1 \mathrm{keV}$ thermal component representing the emission of the LHB, and an absorbed power law with a fixed slope of 1.46 representing the emission from the unresolved AGNs (De Luca \& Molendi 2004). In XSPEC, the thermal emission of the Galactic Halo and the LHB are modelled by the optically thin plasma model apec (Smith et al. 2001) with fixed solar abundances at $z=0$. We used the Asplund et al. (2009) abundance table for the relative abundance of heavy elements. The absorption is given by the Galactic column density of hydrogen, $n_{\mathrm{H}}$, along the line-of-sight of each cluster, and is modelled in XSPEC through phabs. We obtained the $n_{\mathrm{H}}$ value using the method of Willingale et al. (2013, see Table 2).

\subsubsection{Spectral fitting}

The cluster thermal emission is modelled with an absorbed apec model in XSPEC. We re-grouped all XMM-Newton spectra in order to secure at least 25 photon counts per bin, which is necessary when using the $\chi^{2}$ minimization method. We fitted the $X M M-N e w t o n$ spectra in the $0.4-10 \mathrm{keV}$ energy band.

The spectral fitting of the data is done in two steps. First, for the modelling of the CXB we jointly fit the RASS spectra extracted from an annular region (1-2 deg from the cluster position) with XMM-Newton background spectra, with null or minimal cluster residual emission. The RASS spectra are obtained using a modified version of the available tool ${ }^{2}$ at the HEASARC webpage. The main adjustment we did on this tool was to mask out remaining sources in the RASS spectra. The XMM-Newton background spectra are obtained from annular sectors, 12.5-14 arcmin, centred in the observation pointing (see red curves in Figs. 1-3). In this joint fitting, the normalizations of the CXB components are linked across the spectra. The temperature, abundance, and normalization of the cluster residual emission in the XMM-Newton background spectra are only linked across the EPIC detectors. These parameters, together with the normalizations of the residual Gaussian lines, are the only ones we allow to vary in the fitting. We take into account the proper corrections for the observed solid angle in the different spectra.

In a second part of the spectral modelling, we include the spectra extracted from concentric annuli (see white curves in Figs. 1-3) to the joint spectral fitting. The best model parameters of the first fit are taken in this second step as initial values. As before, the normalizations of the CXB components, the normalizations of the residual Gaussian lines, the temperatures, metallicities, normalizations, and when the data allows it, the redshift in each cluster spectra are left free.

\section{Results}

\subsection{Visual impression}

The XMM-Newton images of RXC J2306.6-1319, RXC J0034.6-0208 and ZwCl1665 reveal double or triple extended structures (see Figs. 1-3) centred around these RASS cluster positions. Our main findings of this first exercise are:

- RXC J2306.6-1319. The XMM-Newton observation reveals a double extended structure aligned in the northwest-southeast direction (see Fig. 1). The RXC J2306.6-1319 position is located $\gtrsim 5$ arcmin from the centres of both extended objects. The southeastern extended source is $\sim 1$ arcmin away from

\footnotetext{
2 https://heasarc.gsfc.nasa.gov/cgi-bin/Tools/xraybg/ xraybg.pl
} 


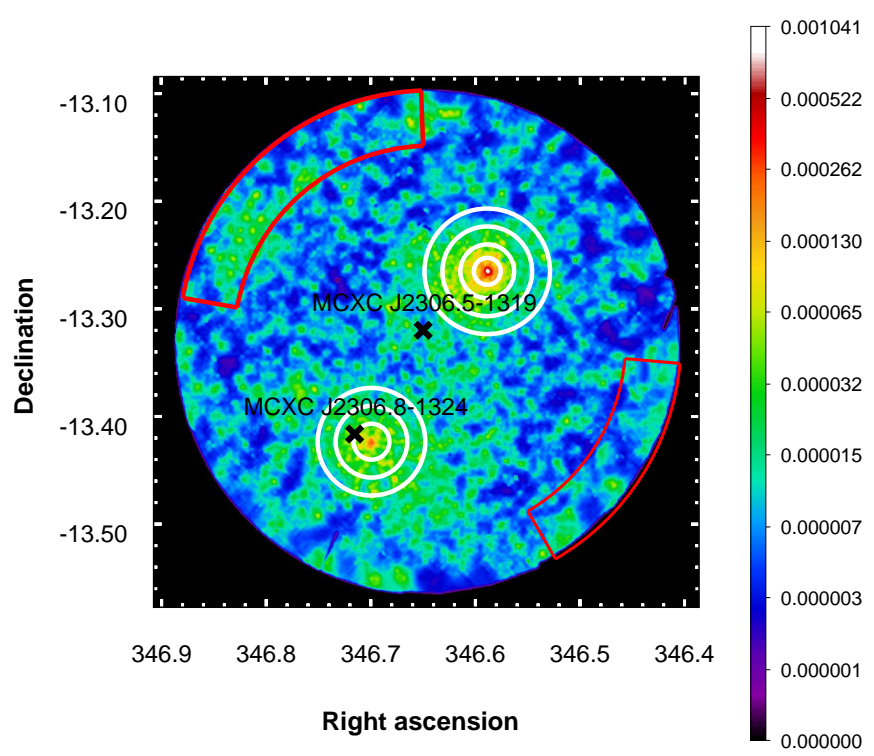

Fig. 1. XMM-Newton smoothed, vignetting-corrected, and backgroundsubtracted image of the RXC J2306.6-1319 observation in the $0.4-1.25 \mathrm{keV}$ energy band. The image is smoothed with a Gaussian kernel of sigma equal to three pixels. The concentric white circles show the chosen regions for the source spectral extraction. The regions delimited by the red curves are used to estimate the local sky background components. The units in the colour bar are count $\mathrm{s}^{-1}$. The black crosses show the location of the MCXC clusters in the field. In this figure, north is up and east to the left.

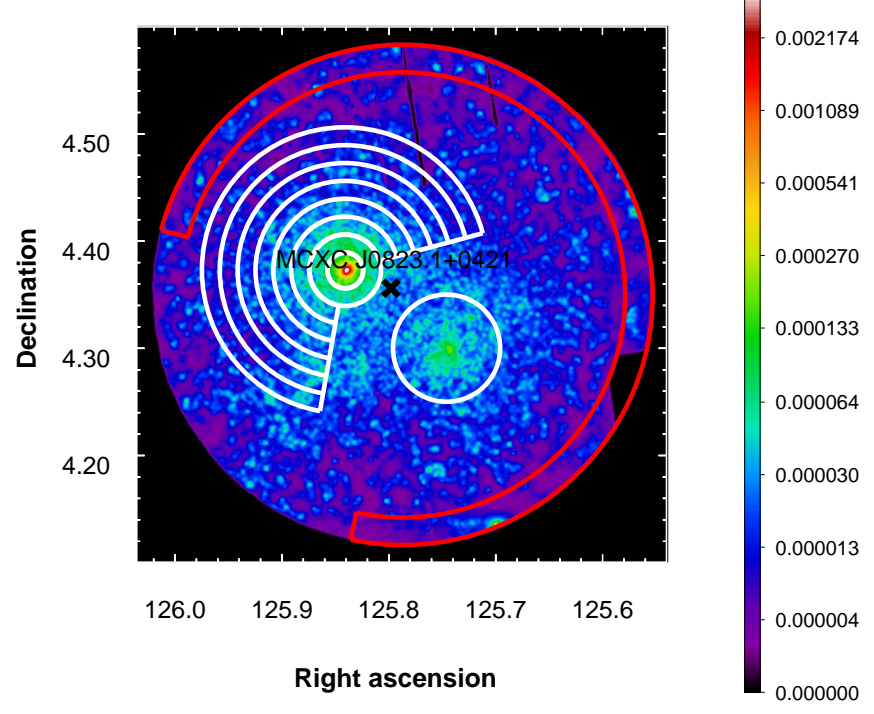

Fig. 2. XMM-Newton smoothed, vignetting-corrected, and backgroundsubtracted image of the $\mathrm{ZwCl} 1665$ observation in the $0.4-1.25 \mathrm{keV}$ energy band. The caption information is the same as for Fig. 1 .

RXC J2306.8-1324. Using the VizieR database (Ochsenbein et al. 2000) we found that one FSC (Faint Source Catalogue, Voges et al. 2000) source is located nearby the southeastern extended component.

- ZwCl 1665. The XMM-Newton observation shows a double extended structure aligned in the northeast-southwest direction (see Fig. 2). The position of $\mathrm{ZwCl} 1665$ is $\sim 2.5$ arcmin from the northeastern objects, while the southwestern component is $\sim 5$ arcmin away. A BSC (Bright Source Catalogue Voges et al. 1999) source coincides with the position of the northeastern

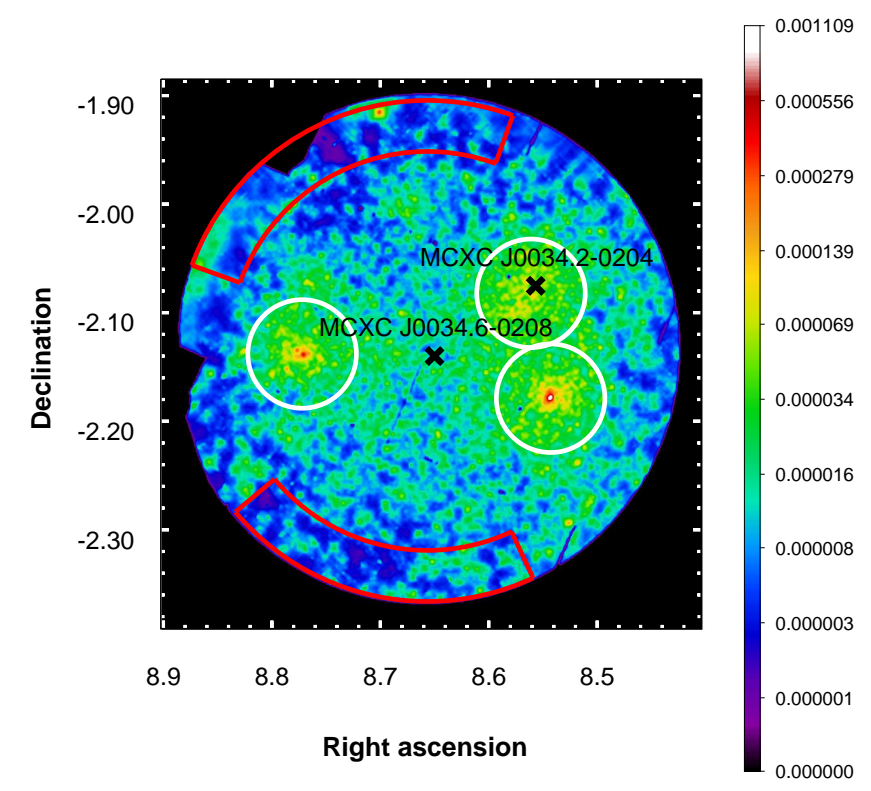

Fig. 3. XMM-Newton smoothed, vignetting-corrected, and backgroundsubtracted image of the RXC J0034.6-0208 observation in the $0.4-1.25 \mathrm{keV}$ energy band. The caption information is the same as for Fig. 1.

structure. Although there are another four FSC sources in the field, none of which seem to be associated with the emission of the southwestern structure.

- RXC J0034.6-0208. The XMM-Newton observation of this cluster shows three extended sources (see Fig. 3). The reported position of RXC J0034.6-0208 lies in the middle of the three extended sources: $\sim 7$ arcmin from the eastern and southwestern components and $\sim 6$ arcmin from the northwestern structure. The position of RXC J0034.2-0204 agrees with an AGN within the northwestern extended source. In this latter case, it would have been difficult to resolve the AGN with ROSAT data. In fact, only one BCS source is in the field, located very close to the southwestern component. The second ROSAT all-sky survey (2RXS) source catalogue (Boller et al. 2016) contains more sources (7) in this field, three of them are located nearby ( $\sim 1-2$ arcmin) each of the extended components.

\subsection{Emission peak, $X$-ray redshift, temperature, and abundance estimation of the cluster components}

We determined the X-ray emission peak of each extended structure in the observations using smoothed background-subtracted and vignetting-corrected images in the $0.4-1.25 \mathrm{keV}$ energy band. Table 3 shows the coordinates of the X-ray peak of each extended structure found in the observations.

We carried out an X-ray analysis for each component in each observation independently. We extracted spectra from concentric annuli of 1 arcmin width (slightly smaller in the case of the northwestern structure in RXC J2306.6-1319), or circular regions centred on the different X-ray structures as shown in Figs. $1-3$ by the white regions. As described in Sect. 3.2.2, we fitted the thermal cluster emission in each region with a thin plasma emission model apec and determined the temperature, metal abundance, and redshift. Since the cluster morphology and data quality are different in each observation, we applied a slightly different spectral fitting approach to each cluster. In the following, we describe the details of the spectral fitting. 
Table 3. Extended structures identified in the different observations.

\begin{tabular}{lcccccc}
\hline \hline Observation & Components & $\begin{array}{c}\text { Identified } \\
\text { groups }\end{array}$ & $\begin{array}{c}\text { RA } \\
(\mathrm{J} 2000)\end{array}$ & $\begin{array}{c}\text { Dec } \\
(\mathrm{J} 2000)\end{array}$ & $z_{\text {spec }}^{\text {opt }}$ & $z_{\text {spec }}^{\text {X-ray }}$ \\
\hline RXC J2306.6-1319 & SE & RXC J2306.8-1324 & 346.699 & -13.424 & $0.068 \pm 0.002$ & $0.065_{-0.009}^{+0.011}$ \\
& NW & A2529 & 346.589 & -13.264 & $0.109 \pm 0.003$ & $0.111_{-0.014}^{+0.011}$ \\
ZwCl 1665 & NE & ZwCl1665 & 125.841 & +4.372 & $0.030 \pm 0.002$ & $0.028_{-0.001}^{+0.002}$ \\
& SW & SDSS-C4-DR3 1283 & 125.746 & +4.300 & $0.097 \pm 0.003$ & $0.106_{-0.032}^{+0.026}$ \\
RXC J0034.6-0208 & E & - & 8.772 & -2.138 & $0.055^{\dagger}$ & $0.045_{-0.012}^{+0.016}$ \\
& NW & RXC J0034.2-0204 & 8.583 & -2.070 & $0.082^{\dagger}$ & $0.028_{-0.007}^{+0.005}$ \\
& SW & - & 8.544 & -2.178 & $0.057^{\dagger}$ & $0.082_{-0.006}^{+0.007}$ \\
\hline
\end{tabular}

Notes. Here, their X-ray peak coordinates, optical spectroscopic redshift, and X-ray spectroscopic redshift are displayed. ${ }^{(\dagger)}$ These redshift values are taken from the closest brightest galaxy to their corresponding X-ray peak.

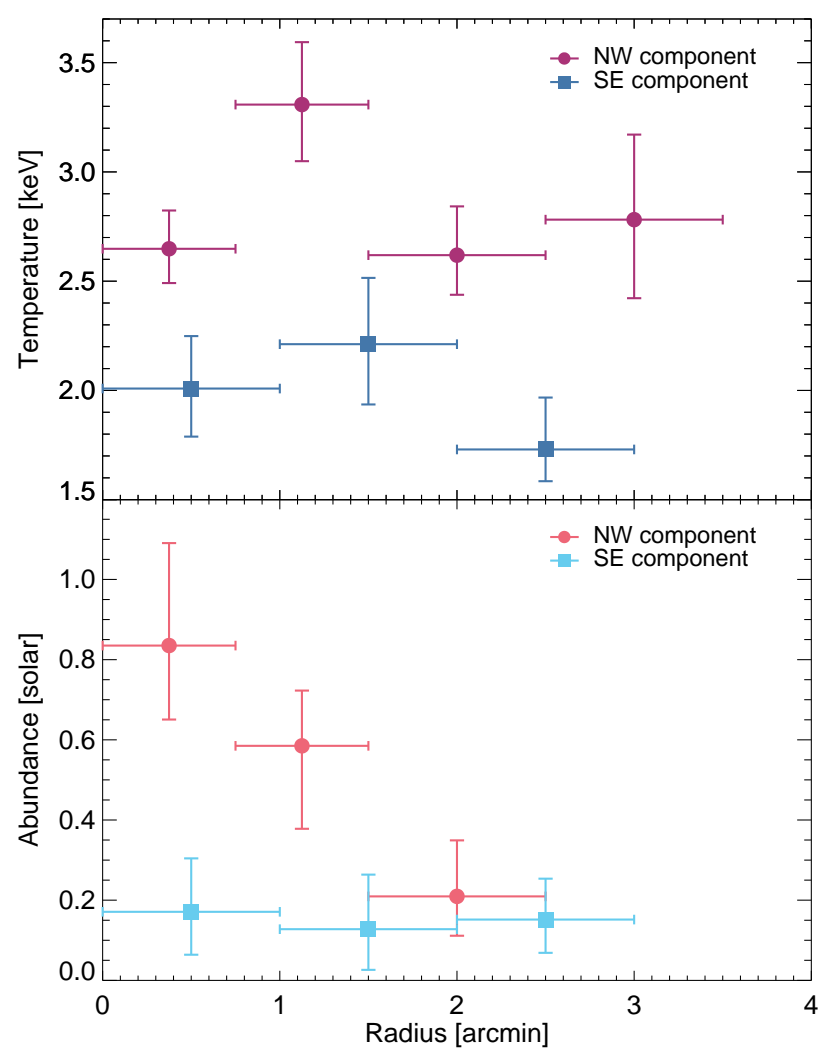

Fig. 4. Temperature (top panel) and metal abundance (bottom panel) profiles for A2529 (northwestern structure in Fig. 1, here in solid circles) and RXC J2306.8-1324 (southeastern source in Fig. 1, here in solid squares). The abundance in the outermost annulus of A2529 has been fixed to a value of 0.15 (see text for details).

- RXC J2306.6-1319. Given the data quality of this observation, we were only able to extract temperature and metal abundance profiles for both clusters for a few annuli: $0-1,1-2$, and $2-3$ arcmin for the southeastern structure, and $0-0.75,0.75-1.50,1.5-2.5$, and $2.5-3.5$ arcmin for the northwestern object (see Fig. 4). The abundance of the outer bin of the northwestern structure could not be constrained, therefore we fixed it to a value of $0.15 Z_{\odot}$, where $Z_{\odot}$ represents the solar abundance. The northwestern structure shows a temperature around $3 \mathrm{keV}$, while the southeastern object has a temperature around $2 \mathrm{keV}$. The temperature profile of the northwestern object exhibits a temperature decrement in the cluster centre, whereas the temperature profile of the southeastern component is consistent with a flat profile. The metal abundance profile of the northwestern structure decreases from $0.8 Z_{\odot}$ in the core to $0.2 Z_{\odot}$. In contrast, the metal abundance of the southeastern object remains flat at a value of $\sim 0.2 Z_{\odot}$. In addition, we could free the redshift parameter during the spectral fitting of the northwestern structure, obtaining $z=0.111_{-0.011}^{+0.014}$. Figure 7 shows the likelihood distribution of this redshift parameter ${ }^{3}$. This redshift value is different from the value of $z=0.066$ reported in Piffaretti et al. (2011) for RXC J2306.6-1319. The redshift in the southeastern structure could not be constrained in this joint spectral fitting; the value of $z=0.066$, which corresponds to RXC J2306.8-1324, was therefore used (Piffaretti et al. 2011). If a circular region of 3 arcmin radius is used for the spectral analysis of the southwestern component, we are able to constrain the redshift $(z=$ $\left.0.065_{-0.009}^{+0.011}\right)$. The redshift difference between the two components is significant at $3 \sigma$.

- ZwCl1665. The data quality of this observation allows us to determine the temperature and metal abundance profiles up to $\sim 8$ arcmin from the cluster centre of the most prominent extended source in the $\mathrm{ZwCl} 1665$ observation (see Fig. 5). As shown in Fig. 2, the spectral extraction regions for the northeastern object avoid the overlapping region with the southwestern component. This cluster also shows a cool-core (CC) feature, that is, the temperature in the innermost region has a lower value than its surroundings. The metal abundance profile exhibits a decreasing behaviour from $0.4 Z_{\odot}$ in the core down to $\sim 0.1 Z_{\odot}$ in the outskirts. For this cluster, we also have enough data to free the redshift value during the spectral fitting. We obtain $z=0.028_{-0.001}^{+0.002}$, which is consistent with the redshift value of ZwCl 1665 reported in Piffaretti et al. (2011). The spectrum of the southwestern structure is heavily contaminated by the emission of the northeastern object, and this must be accounted for in the spectral fitting. In contrast to the previous spectral analysis, the cluster spectral emission of the southwestern structure (within 3 arcmin radius of the X-ray peak, see Fig. 2) is modelled by the background emission plus two apec models, one representing the emission of the northeastern component and the other of the southwestern structure itself. The redshift of the apec model of the northeastern object is kept fixed $(z=0.028)$ since it has been well constrained, and because this reduces the number of free parameters during the fitting process. Moreover, we

3 The likelihood is given by $\mathcal{L}=e^{-\chi^{2} / 2}$, where $\chi^{2}$ is obtained from XSPEC. 


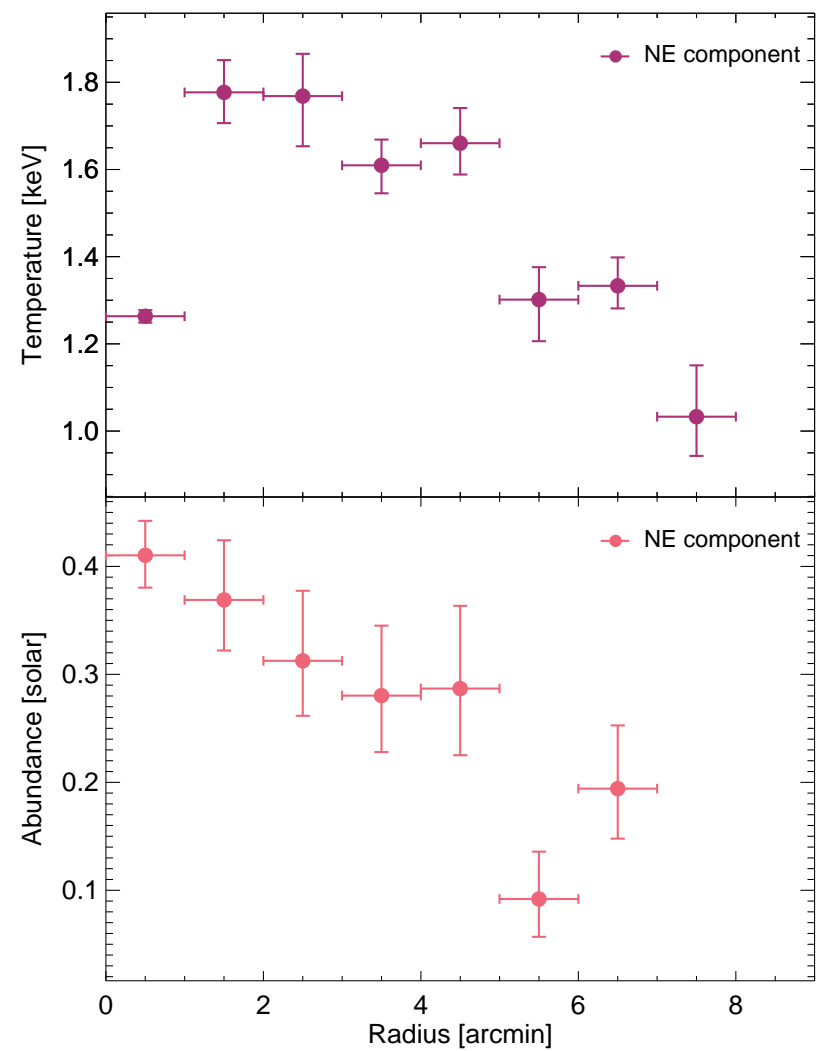

Fig. 5. Temperature (top panel) and metal abundance (bottom panel) profiles of $\mathrm{ZwCl} 1665$. The measurements correspond to the white regions of the northeastern structure in Fig. 2.

include the spectra of a region of the same area as the three last annuli of the northeastern component (5-8 arcmin, see Fig. 2) to help constrain the contaminating emission in the 3 arcmin region of the southwestern source. We use the Markov-chain Monte Carlo (MCMC) method with a Metropolis-Hastings algorithm in XSPEC in order to explore the full parameter space. The length of the MCMC chain is 200000 and we use flat priors. The contaminating emission of the northeastern component on the southwestern structure has a temperature of $k T=1.36_{-0.03}^{+0.04} \mathrm{keV}$ and an abundance of $0.13_{-0.04}^{+0.03} Z_{\odot}$, which are consistent with the values found for the three last individual annuli in the northeastern component (see Fig. 5). The emission of the southwestern structure seems to prefer a high redshift value of $0.106_{-0.032}^{+0.026}$, although this redshift value is consistent within $2.5 \sigma$ with the one of the northeastern component $(z=0.028)$. The results of the parameter sampling for the apec model of the southwestern structure are shown in Fig. 6. Figure 8 also shows the marginalized probability of the redshift. The temperature of the southwestern object is $k T=2.88_{-0.15}^{+0.19} \mathrm{keV}$ and the abundance $0.14_{-0.05}^{+0.12} Z_{\odot}$. When fixing the redshift of the southwestern structure to $z=0.03$ the abundances of the two apec models could not be constrained. The southwestern structure is very faint in the available Chandra data. However, a spectral fitting seems to also favour a redshift $0.1-0.12$.

- RXC J0034.6-0208. The XMM-Newton observation of this cluster is of poor quality (see Sect. 3). Although the emission of the three extended structures in this observation might overlap (as in the case of $\mathrm{ZwCl} 1665$ ), and due to their low photon statistics, we carried out the X-ray analysis for each component independently; that is, we assume no contaminating emission from the two other components while analysing the third one. We extracted spectra for each of the three extended structures within 3 arcmin radius of the respective X-ray peak (see Fig. 3). The joint MOS2 and pn spectral analysis determines the following temperatures, abundances, and redshifts: for the eastern structure we found $k T=1.75_{-0.16}^{+0.10} \mathrm{keV}, Z=0.21_{-0.04}^{+0.05} Z_{\odot}$, and $z=$ $0.045_{-0.012}^{+0.016}$, for the northwestern structure, $k T=2.45_{-0.14}^{+0.09} \mathrm{keV}$, $Z=0.50_{-0.08}^{+0.06} Z_{\odot}$, and $z=0.028_{-0.005}^{+0.007}$, and for the southwestern structure $k T=2.09_{-0.07}^{+0.07} \mathrm{keV}, Z=0.42_{-0.06}^{+0.04} Z_{\odot}$, and $z=$ $0.082_{-0.006}^{+0.007}$. Only two of the three components agree within $2 \sigma$ with the reported redshift values $(z \sim 0.08$, Piffaretti et al. 2011) of RXC J0034.6-0208 and RXC J0034.2-0204 clusters. Given the short XMM-Newton observation it is difficult to detect the iron $\mathrm{K}$ and $\mathrm{L}$ lines of each component, which might lead to these differences in redshift. In Appendix A we explore these differences further by analysing each detector separately.

\subsection{Database search and optical redshift analysis}

We combined the available information from the NASA/IPAC Extragalactic Database ${ }^{4}$ (NED) and SDSS DR $14^{5}$ to construct spectroscopic redshift distributions of each observation (see Figs. 7-9). For this, we select all galaxies with spectroscopic redshift within 30 arcmin of the MCXC cluster positions. All the individual redshift distributions of these observations reveal two structures (bi-modal distributions) at different redshifts. In these distributions, one of the peaks is consistent with the MCXC redshift (see Table 1).

We fit a Gaussian function to each of the peaks in the redshift distribution to obtain a redshift estimation of the different structures. For the structures in RXC J2306.6-1319 and ZwCl 1665, these values are displayed in Table 3 under the column labelled $z_{\text {spec }}^{\text {opt }}$, and they are consistent (within $1 \sigma$ ) with the one obtained from our X-ray analysis (column $z_{\text {spec }}^{\mathrm{X} \text {-ray }}$ also in Table 3).

We further investigate these extended structures by searching in NED for known galaxy groups and/or clusters with available spectroscopic redshift information within 15 arcmin of each observation. Our findings are summarised as follows.

- RXC J2306.6-1319. The northwestern object is located nearby A2529 ( $<1$ arcmin). The redshift of this cluster is $z=$ 0.111 (Struble \& Rood 1999), which is in agreement with the redshift obtained from our X-ray spectroscopic analysis.

$-\mathrm{ZwCl} 1665$. Within $\sim 6$ arcmin of the northeastern object position, NED shows several galaxy groups and clusters with spectroscopic redshifts of $z \sim 0.03$, which agree with the value obtained from our X-ray spectroscopic analysis. One of these groups is SDSS-C4-DR3 $1356(z=0.03$ from 34 galaxies, Von Der Linden et al. 2007). The location of the southwestern structure agrees $(<0.1$ arcmin) with the SDSS-C4-DR3 1283 cluster position $(z=0.095$ from 23 galaxies, Von Der Linden et al. 2007). This latter extended substructure is also located $\sim 1$ arcmin away from a cluster in the recently published galaxy cluster catalogue by Banerjee et al. (2018) (ID 10959 with $z=0.0958)$. This redshift is also in agreement with the redshift obtained from our X-ray analysis.

- RXC J0034.6-0208. NED does not show other galaxy groups or clusters besides RXC J0034.6-0208 and RXC J0034.2-0204. The X-ray peak positions of the eastern and southwestern extended components coincide with bright elliptical galaxies (see Fig. 12) with $z \sim 0.055$ (from SDSS DR14). On the contrary, the X-ray peak of the northwestern

\footnotetext{
4 https://ned.ipac.caltech.edu/

http://wWw. sdss.org/dr14/
} 


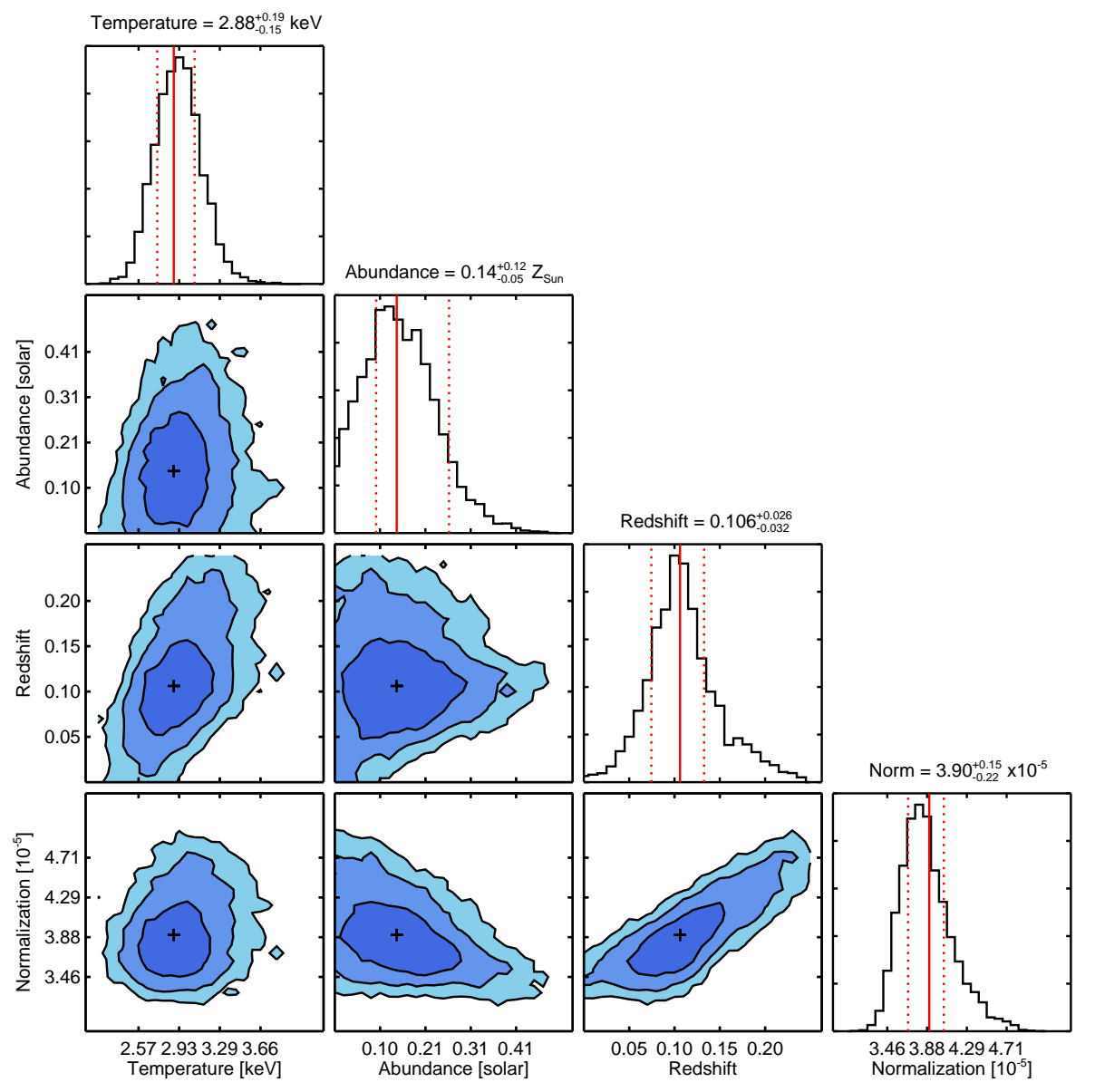

Fig. 6. Marginalized 2D and 1D constraints on the apec model parameters obtained through an MCMC in XSPEC of the southwestern structure in the $\mathrm{ZwCl} 1665$ observation. The colours in the $2 \mathrm{D}$ plots represent the 68,95 , and $99 \%$ credibility intervals. The red solid line on top of the 1D constraints and the cross overlaid on the 2D contours indicate the best-fitting XSPEC values, the red dotted lines show the $68 \%$ confidence interval.

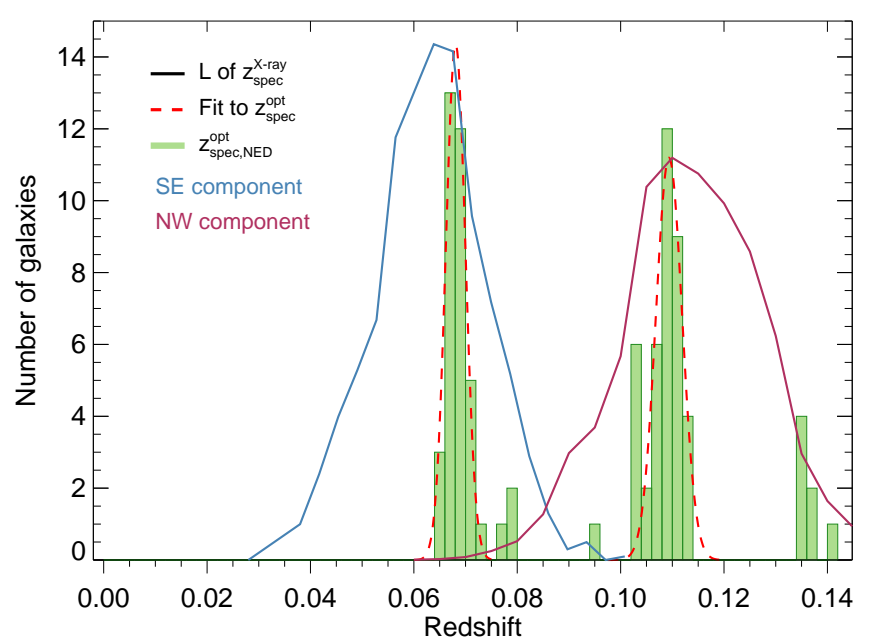

Fig. 7. Spectroscopic redshift histogram for galaxies within $30 \mathrm{arcmin}$ of the RXC J2306.6-1319 cluster position. The peaks of this distribution are fitted with Gaussian functions (red dashed lines), obtaining $z=0.068 \pm 0.002$ for the low-redshift peak and $z=0.109 \pm 0.003$ for the high-redshift structure. The solid lines show the likelihood distribution of the redshift parameter obtained from the joint X-ray spectral fitting of the two structures shown in Fig. 1. The blue line shows the southeastern component, while the purple one the northwestern object.

component is located nearby an elliptical galaxy with a spectroscopic redshift value of $z=0.082$. The redshifts of these bright elliptical galaxies are displayed in Table 3. Only the redshift of the brightest galaxy in the eastern structure is in agreement within $1 \sigma$ with the redshift obtained our X-ray analysis $(z \sim 0.045)$.

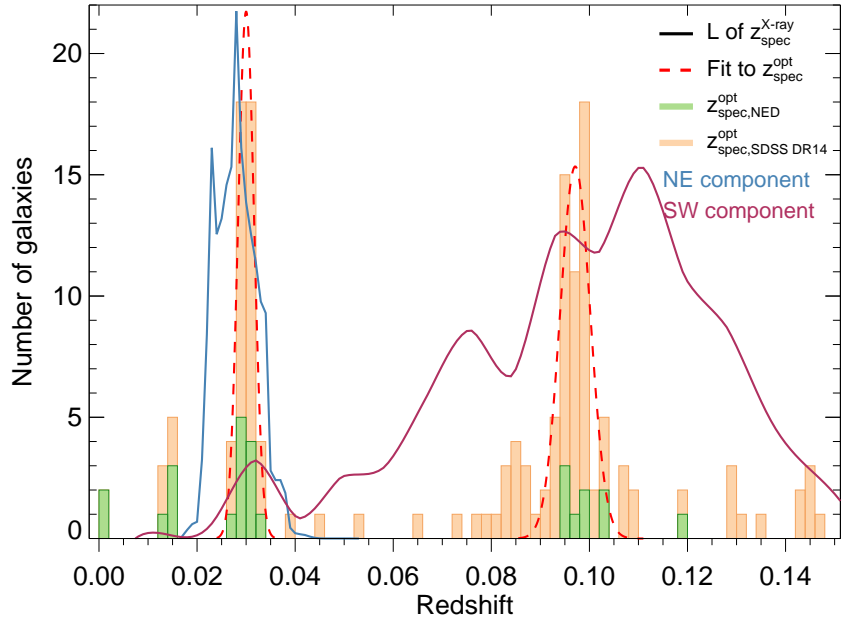

Fig. 8. Spectroscopic redshift histogram for galaxies within 30 arcmin of the $\mathrm{ZwCl} 1665$ cluster position. The caption information is the same as for Fig. 7, with the addition that the orange histogram shows the number of galaxies with available SDSS DR14 redshifts. The Gaussian fit of the low-redshift peak gives $0.030 \pm 0.002$, while for the highredshift one we obtain $0.097 \pm 0.003$. The likelihood distributions of the redshift parameter of the northeastern component is in purple, while the southwestern object is shown in blue.

We applied a $3 \sigma$ clipping to each redshift structure in their distributions to remove outliers, and display the projected distribution of the galaxies (see Figs. 10-12). Although the redshift information is not complete, it can give us a good idea of the galaxy redshift distribution in the plane of the sky. Figure 10 shows a projected redshift separation in the RXC J2306.6-1319 


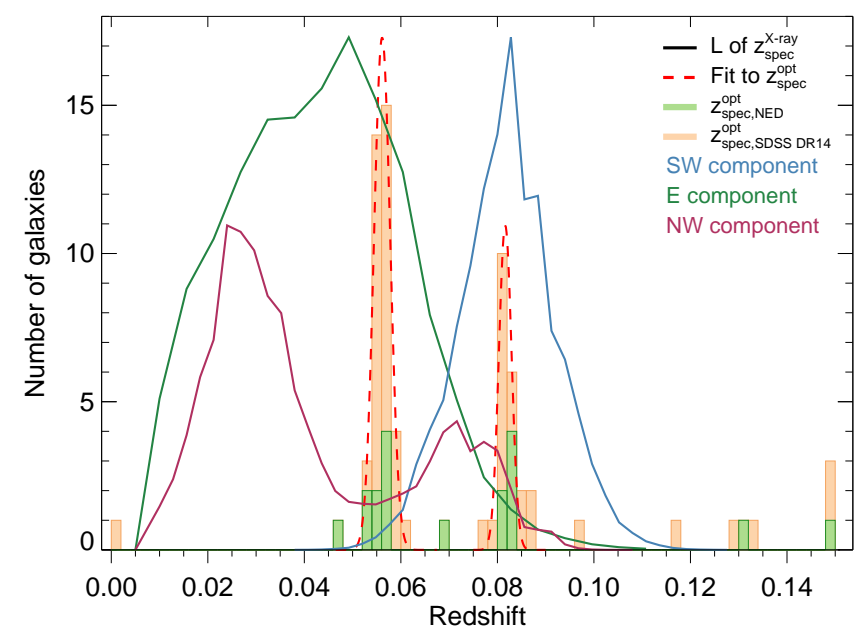

Fig. 9. Spectroscopic redshift histogram for galaxies within $30 \mathrm{arcmin}$ of the RXC J0034.6-0208 cluster position. The caption information is the same as for Fig. 8. The Gaussian fit of the low-redshift peak gives $z=0.056 \pm 0.002$, while the high-redshift structure peaks at $z=0.082 \pm 0.001$. The likelihood distributions are in green the eastern component, in purple the northwestern object, and in blue the southwestern structure.

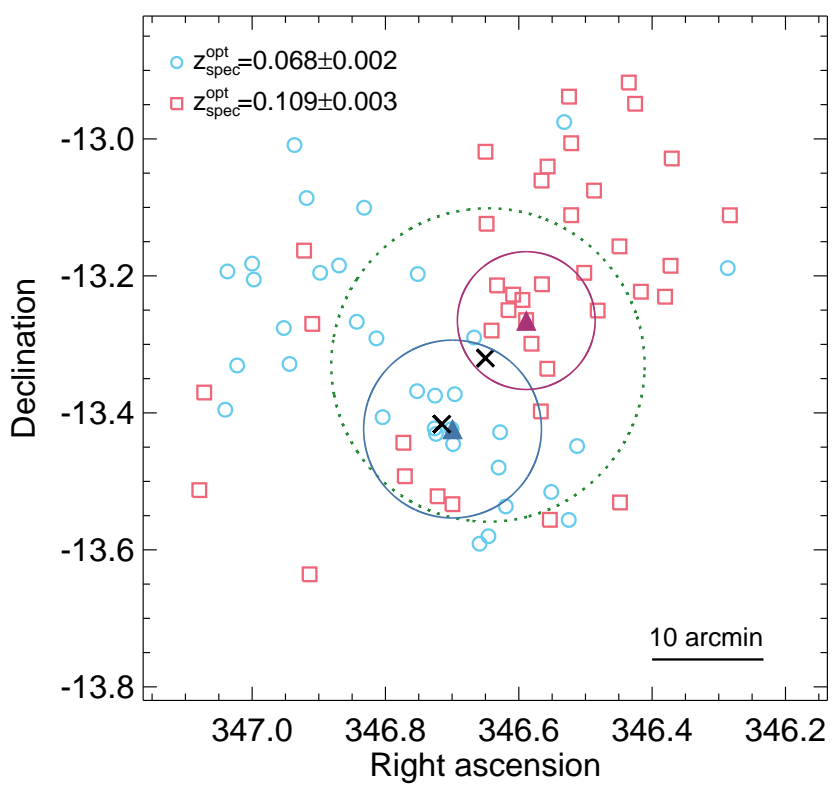

Fig. 10. Projected redshift distribution of galaxies with spectroscopic redshift information for the RXC J2306.6-1319 observation. Light-blue circles correspond to galaxies with redshift values of $\sim 0.068$, while pink squares show galaxies with $\sim 0.109$. The black crosses correspond to the position of the MCXC clusters in the observation and the green dotted circle shows the FoV of XMM-Newton (see Fig. 1 for comparison). The purple filled triangle marks the X-ray peak position of A2529, while the blue one of RXC J2306.8-1324. Their corresponding circles show $r_{500}$. In this figure, north is up and east to the left.

region, with a higher concentration of galaxies with $z \sim 0.109$ in the northwest direction. The case of $\mathrm{ZwCl} 1665$ is not as clear as RXC J2306.6-1319; galaxies of the two redshifts estimations cover the whole plane of the sky, however there is a slight over-density of galaxies with $z=0.097 \pm 0.003$ close to the position of SDSS-C4-DR3 1283. Around the eastern and southwestern components of RXC J0034.6-0208 we can see a higher concentration of galaxies around $z \sim 0.056$, while the northwestern structure lies close by to galaxies with $z \sim 0.082$.

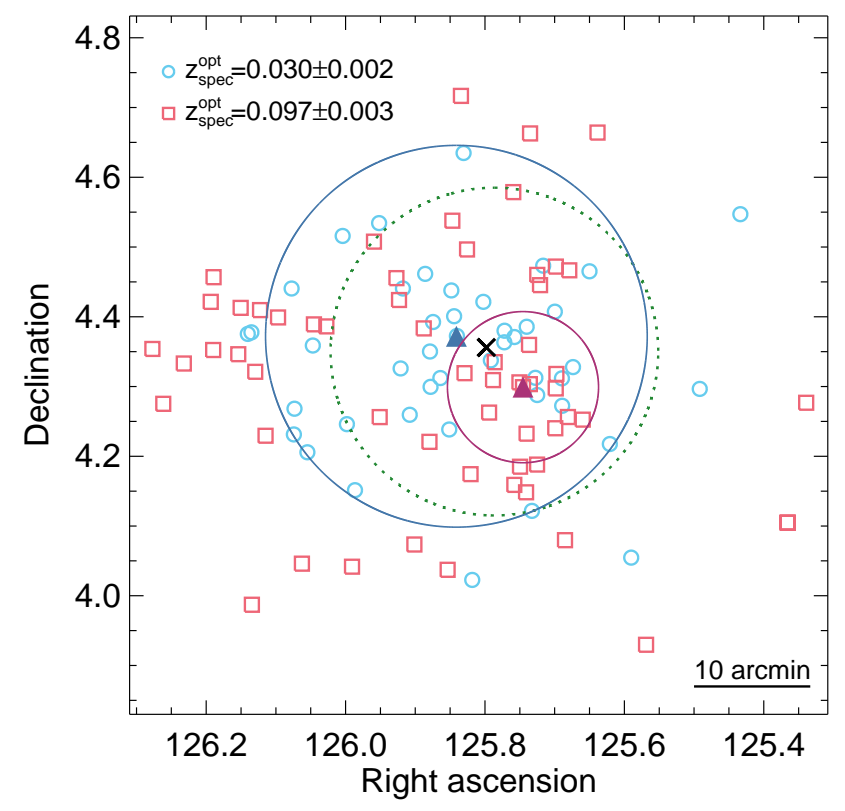

Fig. 11. Projected redshift distribution of galaxies with spectroscopic redshift information for the $\mathrm{ZwCl} 1665$ observation. The caption information is the same as for Fig. 10, with the exception that light-blue circles correspond to galaxies with redshift values of $\sim 0.030$ and pink squares show galaxies with $\sim 0.097$. The purple and blue filled triangles show the X-ray peak positions of $\mathrm{ZwCl} 1665$ and SDSS-C4-DR3 1283, respectively.

Given the results of the optical spectroscopic redshift and $\mathrm{X}$-ray spectral analysis, we relate the southeastern and northwestern structures in the observation RXC J2306.6-1319 to RXC J2306.8-1324 and A2529, respectively. In the same way, we connect the southwestern extended component in $\mathrm{ZwCl} 1665$ to cluster SDSS-C4-DR3 1283. The case of RXC J0034.6-0208 is more complex, given that the X-ray spectral analysis seems to give contradicting redshift information in comparison with the optical data.

\subsection{Global properties}

\subsubsection{Mass and radius}

In this section we derive the global properties of each galaxy cluster namely the emission weighted global temperature, $k T$, the global metallicity, $Z$, the mass, $M_{500}$, and the corresponding radius, $r_{500}$, flux, $F_{500}$, and luminosity, $L_{500}$.

For some galaxy groups and clusters the gas temperature, $k T$, decreases in the central regions, resulting in CC systems. In order to avoid the effect of $\mathrm{CC}$, that is, biasing the global gas temperature estimate low, for clusters with enough photon counts and with good-data quality we measure the global gas temperature within $0.1-0.5 r_{500}$ (Zhang et al. 2006); for the other clusters the temperature is determined within $0.5 r_{500}$ (see Table 4). To estimate $M_{500}$ we use the scaling relation obtained by Lovisari et al. (2015):

$\log _{10}\left(\frac{M_{500}}{5 \times 10^{13} M_{\odot}}\right)=1.65 \log _{10}\left(\frac{k T}{2 \mathrm{keV}}\right)+0.19$.

We choose this scaling relation since it has been determined using a galaxy group sub-sample of eeHIFLUGCS with similar temperatures to the ones we find in this work. An initial temperature is obtained by performing a spectral fitting to the data in a given aperture. The global gas temperature and $M_{500}$ 

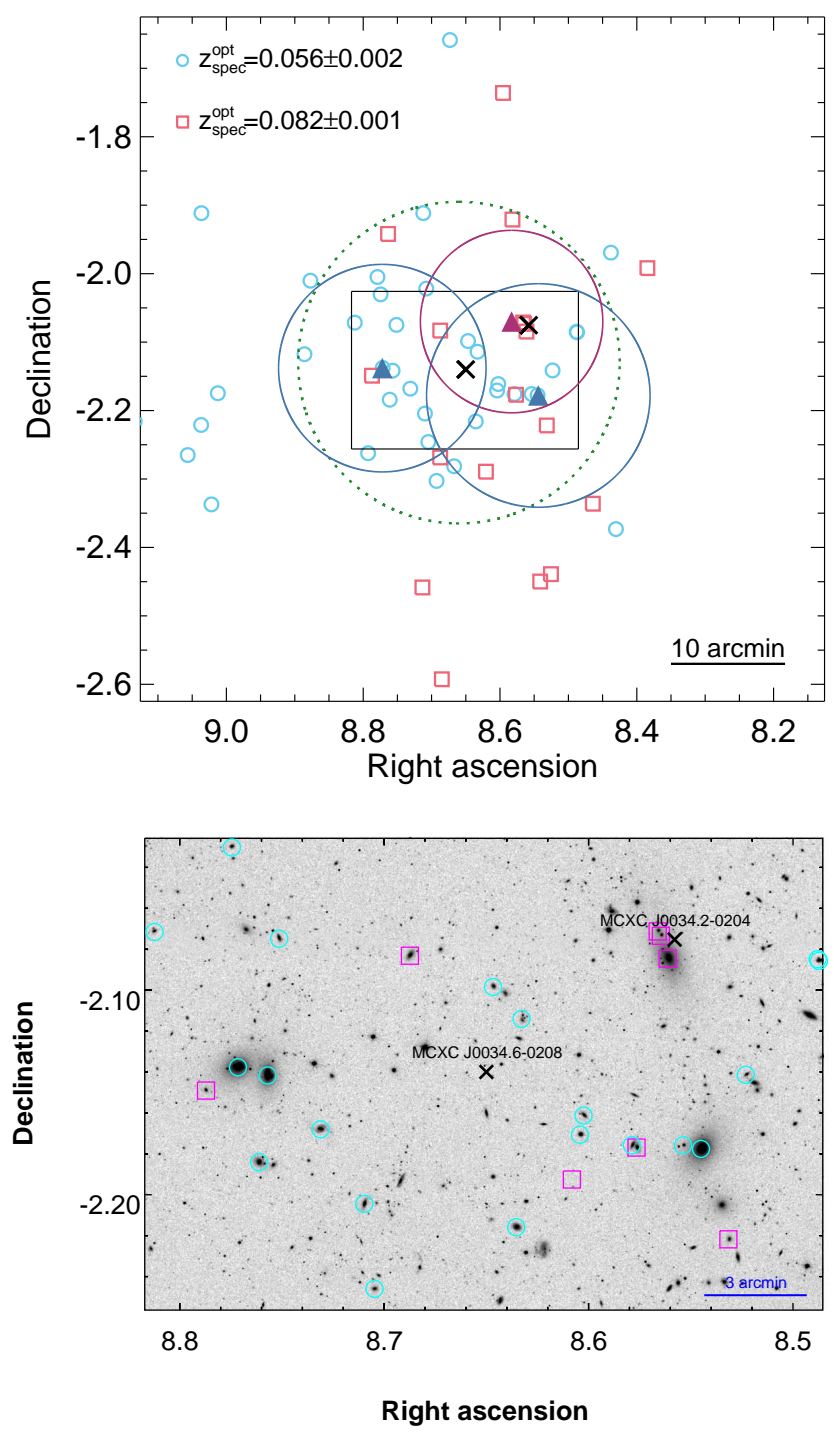

Fig. 12. Top: projected redshift distribution of galaxies with spectroscopic redshift information for the RXC J0034.6-0208 observation. The caption information is the same as for Fig. 10, with the exception that light-blue circles correspond to galaxies with redshift values of $\sim 0.056$ and pink squares show galaxies with $\sim 0.082$. The filled triangles shows the X-ray peak positions of the three extended substructures in the field. Bottom: SDSS $r$-band image of the region delimited by the black solid line in the top panel.

are evaluated iteratively until we obtain a stable temperature. We determine $r_{500}$ from $M_{500}$ using

$r_{500}=\left(\frac{M_{500}}{\frac{4 \pi}{3} 500 \rho_{\mathrm{cr}}(z)}\right)^{\frac{1}{3}}$,

where $\rho_{\mathrm{cr}}(z)$ is the critical density at the cluster redshift. The estimated $k T, Z, M_{500}$ and $r_{500}$ of each galaxy cluster studied in this work are listed in Table 4 . We notice that four of the analysed groups in this work have abundance values $Z \lesssim 0.2 Z_{\odot}$, either within $0.5 r_{500}$ or $0.1-0.5 r_{500}$. In a study of $\sim 200$ galaxy groups and clusters, Lovisari \& Reiprich (2019) found such low metallicities for only one or two objects within $0.3 r_{500}$.

\subsubsection{Flux and luminosity}

Given that most of the newly identified sub-clusters overlap in the plane of the sky, we needed to fit their surface-brightness

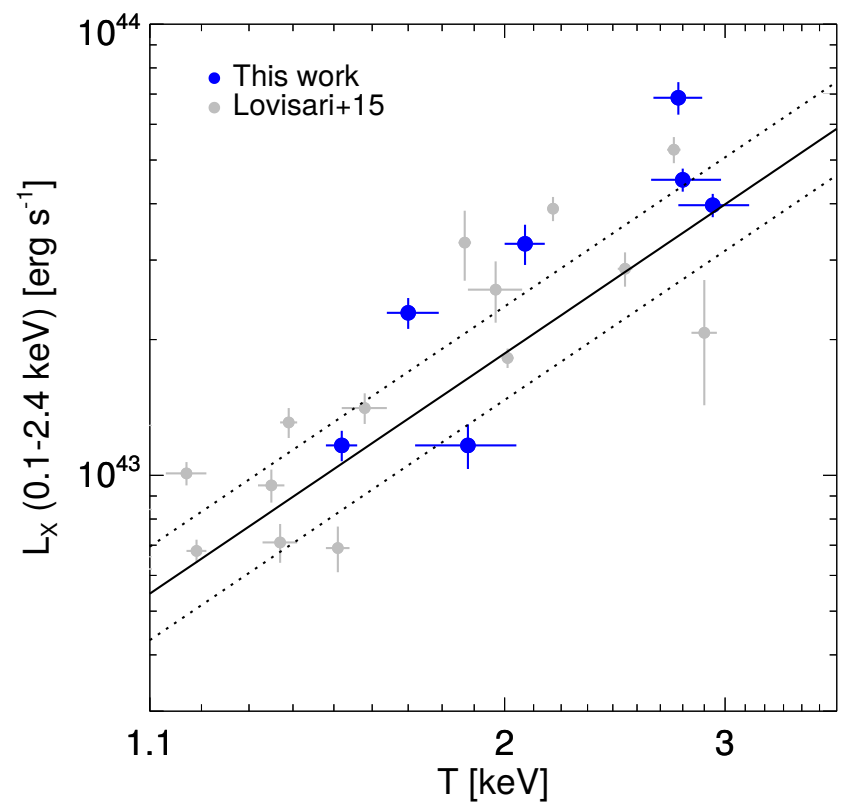

Fig. 13. Comparison of the global properties, luminosity, and temperature of the analysed clusters in this work (blue circles) with the group sample studied in Lovisari et al. (2015) (grey points). The solid/dashed blue lines correspond to the non-bias-corrected $L_{\mathrm{X}}-T$ scaling relation from the same authors and its total scatter.

profiles simultaneously to estimate their individual fluxes. We chose the $0.4-1.25 \mathrm{keV}$ energy band for this, as the cluster signal vastly dominates over the background in this soft band, while the absence of strong fluorescence lines in the instrumental background ensure minimal systematic errors - $\mathrm{Al} \mathrm{K} \alpha$ and $\mathrm{Si}$ $\mathrm{K} \alpha$ at $\sim 1.5$ and $\sim 1.7 \mathrm{keV}$, respectively, prevented us from using higher energies. Each of the two or three sub-clusters are modelled as a set of concentric annuli of 3 arcsec in width whose $0.4-1.25 \mathrm{keV}$ surface brightnesses are simultaneously fitted. The position- and instrument-dependent XMM-Newton point spread function is taken into account during the fit. For a set of parameters, we used the best-fit spectral model from the results discussed in Sect. 4.2 to convert the flux profile into count-rates for each instrument. We combined these with a sky background countrate also derived from our best-fit spectral model, the exposure map, and template-based instrumental background maps to form a counts map. Poisson statistics over the whole of the images is then used to derive the best-fit surface-brightness profiles. Statistical uncertainties are finally determined using a Metropolis MCMC analysis based on the covariance of 100000 element chains.

We determine the flux in $r_{500}$ for each cluster by integration of the surface-brightness profiles within the radii reported in Table 4 (all components can be traced out to $r_{500}$ ). The statistical uncertainties are combined in quadrature with the errors on the background flux in the same aperture obtained from our spectral modelling. Finally, the cluster optical redshift and bestfit model is used to convert this flux into a luminosity, ignoring the uncertainty on the cluster spectral model in the process.

The measured $f_{500}$ and $L_{500}$ in the $0.1-2.4 \mathrm{keV}$ energy band, respectively, for each cluster is displayed in Table 4.

Our flux measurements show that out of all the individual systems studied in this work only the northeastern component in the $\mathrm{ZwCl} 11665$ observation has a flux value high enough $(5.7 \times$ $10^{-12} \mathrm{erg} \mathrm{s}^{-1} \mathrm{~cm}^{-2}$ ) to be kept in eeHIFLUGCS (see Sect. 2).

Figure 13 compares our luminosity and temperature measurements with the ones of Lovisari et al. (2015) group sample. We 
Table 4. Derived global properties for the galaxy groups.

\begin{tabular}{lcccccc}
\hline \hline Cluster & $\begin{array}{c}k T \\
(\mathrm{keV})\end{array}$ & $\begin{array}{c}Z \\
\left(Z_{\odot}\right)\end{array}$ & $\begin{array}{c}r_{500} \\
(\mathrm{kpc})\end{array}$ & $\begin{array}{c}M_{500} \\
\left(10^{13} M_{\odot}\right)\end{array}$ & $\begin{array}{c}f_{500} \\
\left(10^{-12} \mathrm{erg} \mathrm{s}^{-1} \mathrm{~cm}^{-2}\right)\end{array}$ & $\begin{array}{c}L_{500} \\
\left(10^{43} \mathrm{erg} \mathrm{s}^{-1}\right)\end{array}$ \\
\hline RXC J2306.8-1324 $^{\dagger}$ & $1.88_{-0.16}^{+0.16}$ & $0.21_{-0.07}^{+0.09}$ & $625_{-18}^{+42}$ & $6.98_{-0.98}^{+1.07}$ & $1.05 \pm 0.12$ & $1.17 \pm 0.13$ \\
A2529 $^{\dagger}$ & $2.70_{-0.14}^{+0.18}$ & $0.38_{-0.11}^{+0.08}$ & $752_{-13}^{+41}$ & $12.73_{-1.32}^{+1.45}$ & $1.54 \pm 0.09$ & $4.52 \pm 0.27$ \\
ZwCl 1665 $^{\dagger}$ & $1.52_{-0.04}^{+0.04}$ & $0.20_{-0.02}^{+0.02}$ & $554_{-11}^{+13}$ & $4.93_{-0.31}^{+0.34}$ & $5.68 \pm 0.44$ & $1.17 \pm 0.09$ \\
SDSS-C4-DR3 1283* $^{*}$ & $2.84_{-0.18}^{+0.16}$ & $0.16_{-0.07}^{+0.08}$ & $757_{-29}^{+27}$ & $13.80_{-1.45}^{+1.61}$ & $1.73 \pm 0.10$ & $3.97 \pm 0.24$ \\
RXC J0034.6-0208 E* $^{*}$ & $1.70_{-0.06}^{+0.09}$ & $0.16_{-0.03}^{+0.03}$ & $582_{-17}^{+16}$ & $5.92_{-0.50}^{+0.53}$ & $3.22 \pm 0.25$ & $2.29 \pm 0.18$ \\
RXC J0034.6-0208 NW $^{*}$ & $2.68_{-0.11}^{+0.11}$ & $0.52_{-0.09}^{+0.10}$ & $742_{-20}^{+21}$ & $12.55_{-1.01}^{+1.10}$ & $4.24 \pm 0.35$ & $6.87 \pm 0.57$ \\
RXC J0034.6-0208 SW $^{*}$ & $2.07_{-0.07}^{+0.07}$ & $0.30_{-0.04}^{+0.05}$ & $648_{-15}^{+16}$ & $8.17_{-0.56}^{+0.62}$ & $4.27 \pm 0.44$ & $3.26 \pm 0.33$ \\
\hline
\end{tabular}

Notes. The flux, $f_{500}$, and luminosity, $L_{500}$, are obtained in the $0.1-2.4 \mathrm{keV}$ observer and rest-frame energy band, respectively. ${ }^{\left({ }^{\dagger}\right)}$ Global temperature determined within $0.1-0.5 r_{500} \cdot{ }^{(*)}$ Global temperature determined within $0.5 r_{500}$.

Table 5. Comparison of fluxes in the $0.1-2.4 \mathrm{keV}$ energy band (see text for details).

\begin{tabular}{lccc}
\hline \hline Cluster & $f_{500}^{\mathrm{MCXC}}$ & $\begin{array}{c}f_{500}^{\mathrm{GCA}} \\
\left(10^{-12} \mathrm{erg} \mathrm{s}^{-1} \mathrm{~cm}^{-2}\right)\end{array}$ \\
\hline RXC J2306.6-1319 & 5.58 & $5.55 \pm 1.00$ & $2.59 \pm 0.15$ \\
ZwCl 1665 & 9.81 & $5.80 \pm 1.10$ & $7.40 \pm 0.45$ \\
RXC J0034.6-0208 & 7.95 & $8.24 \pm 0.71$ & $11.7 \pm 0.62$ \\
\hline
\end{tabular}

chose this sample because Lovisari et al. (2015) obtained its measurements using XMM-Newton observations as well, and the procedures for determining each observable are similar to the ones used in this work. The corresponding non-bias-corrected relation from that work is also shown in Fig. 13, together with its total scatter on the luminosity ( $\sim 20 \%)$. Taking into account the total scatter, the agreement is good despite that the group sample of Lovisari et al. (2015) covers only lower redshift objects $(z<0.035)$.

Finally, we compare the XMM-Newton determined fluxes for the different cluster systems with the overall flux estimation from ROSAT data. With this, we aim to examine the reliability of the previous flux measurements. To do so, we compare three quantities: the flux expected from the MCXC catalogue using the redshift information from MCXC, the ROSAT flux estimation using the growth curve analysis (GCA), and the total flux from XMM-Newton using $z_{\text {spec }}^{\text {opt }}$ in Table 3. The MCXC fluxes, $f_{500}^{\mathrm{MCXC}}$, are estimated using the luminosities and redshifts from $\mathrm{MCXC}$, together with the luminosity-temperature, $L_{\mathrm{X}}-T$ scaling relation of Reichert et al. (2011). To relate observer frame and rest frame quantities, we apply a $k$-correction assuming an abundance of $0.3 Z_{\odot}$ (Asplund et al. 2009). The CGA fluxes, $f_{500}^{\mathrm{GCA}}$, are obtained using a similar photometric analysis like the one described in Böhringer et al. (2000, 2001), and it is fully described in $\mathrm{Xu}$ et al. (2018). In our case, this method integrates the background subtracted count rate in the ROSAT hard band $(0.5-2 \mathrm{keV})$ up-to $r_{500}$. The total flux from XMM-Newton, $f_{500}^{X M M}$, is obtained by adding the individual fluxes (Table 4) of the corresponding components in the cluster systems. Table 5 shows the values of the different estimated fluxes. There is good agreement between the $f_{500}^{\mathrm{MCXC}}$ and $f_{500}^{\mathrm{GCA}}$ measurements for the RXC J2306.6-1319 and RXC J0034.6-0208 systems, which indicates that our method is consistent with the one in Böhringer et al. $(2000,2001)$. However, the $f_{500}^{X M M}$ values of these systems differ from those measurements. This contrasts with the good agreement between the $L_{500}$ values from
MCXC (see Table 1) and the total XMM-Newton luminosity, that is, when adding the individual $X M M$-Newton luminosities we obtain $\sim 5.7$ and $\sim 12.4 \times 10^{43} \mathrm{erg} \mathrm{s}^{-1}$ for RXC J2306.6-1319 and RXC J0034.6-0208, respectively (see Table 4). The difference between ROSAT and XMM-Newton fluxes could be due to different redshift and modelling assumptions. We mainly attribute the disagreement between $f_{500}^{\mathrm{GCA}}$ and $f_{500}^{X M M}$ fluxes for these two systems to the different redshift and modelling assumptions used to characterise each individual object. While for $f_{500}^{\mathrm{GCA}}$ the MCXC redshift is used, the $f_{500}^{X M M}$ is obtained from systems at different redshifts. Moreover, the flux discrepancy can be partially justified in system RXC J2306.6-1319 by better detection of point sources in the XMM-Newton data. However, this does not explain that $f_{500}^{X M M}$ is larger than $f_{500}^{\mathrm{GCA}}$ in the other two systems.

The $f_{500}^{\mathrm{GCA}}$ and $f_{500}^{X M M}$ values for $\mathrm{ZwCl} 1665$ agree within $1.5 \sigma$, however the value of $f_{500}^{\mathrm{MCXC}}$ is higher than those values. The parent catalogue of $\mathrm{ZwCl} 1665$ is BCS from Ebeling et al. (1998), who uses a Voronoi Tessellation and Percolation (VTP) method to estimate the count-rate and extent of the clusters in their catalogue. For this particular cluster, they obtain a higher count-rate in a smaller extraction radius. These differences with respect to the GCA method may explain the difference of a factor of approximately two between the $f_{500}^{\mathrm{GCA}}$ and $f_{500}^{\mathrm{MCXC}}$ for $\mathrm{ZwCl} 1665$.

\section{Discussion and conclusion}

The XMM-Newton and Chandra follow-up of the eeHIFLUGCS sample of galaxy clusters has revealed that three clusters are the result of projection effects of two (or three) physically independent clusters at different redshifts. Our X-ray spectral analysis reveals that the redshift of at least one of the components in each of these multiple systems has a different redshift from the one reported in the literature (Piffaretti et al. 2011). The measured $\mathrm{X}$-ray redshifts are confirmed by a spectroscopic optical analysis, which shows bi-modal redshift distributions in the sky region of the analysed clusters. We report on the global properties of each component: the gas temperature, the total masses within $r_{500}$, the flux, and the luminosity.

In the RXC J2306.6-1319 observation, we found two clusters at redshifts $z \sim 0.06$ and $z \sim 0.11$. The cluster at lower redshift is consistent in position and redshift with the RXC J2306.8-1324 cluster reported by Cruddace et al. (2002) in the SGP catalogue. Our analysis reveals that the higherredshift cluster is in location and redshift agreement with A2529. RXC J2306.8-1324 is less massive $\left(M_{500} \sim 7 \times 10^{13} M_{\odot}\right)$ 
than A2529 $\left(M_{500} \gtrsim 1.2 \times 10^{14} M_{\odot}\right)$. Our surface-brightness analysis shows that both clusters have fluxes below the eeHIFLUGCS flux limit $\left(5 \times 10^{-12} \mathrm{erg} \mathrm{s}^{-1} \mathrm{~cm}^{-2}\right)$, therefore both clusters are no longer part of this cluster sample. Moreover, RXC J2306.6-1319 appears in the supercluster sample of Chon et al. (2013), with a multiplicity of two, that is, the number of clusters that form the supercluster at $z=0.067$. This supercluster sample is based on the REFLEX II catalogue (Böhringer et al. 2013), which is an extension of REFLEX with a lower flux limit. If indeed, Böhringer et al. (2013) included RXC J2306.8-1324 in REFLEX II (the REFLEX II is not publicly available), then this cluster together with RXC J2306.6-1319 would resemble a supercluster. However, our findings show that RXC J2306.6-1319 is indeed a single cluster at higher redshift.

The X-ray spectral analysis of the two extended components in the $\mathrm{ZwCl} 1665$ observation also shows that both structures are located at different redshifts $(z \sim 0.03$ and $z \sim 0.10)$. ZwCl 1665 is the lower-redshift galaxy group, and is confirmed by several optical counterparts. ZwCl 1665 is a cool system $(k T \sim 1.5 \mathrm{keV})$ with a mass of $M_{500} \sim 5 \times 10^{13} M_{\odot}$. The second group identified in the $\mathrm{ZwCl} 1665$ observation has a lower flux due to its higher redshift but is hotter $(\sim 3 \mathrm{keV})$, and more massive $\left(M_{500} \gtrsim 1.3 \times 10^{14} M_{\odot}\right)$ than $\mathrm{ZwCl} 1665$. We cross-identified this system with the SDSS-C4-DR3 1283 cluster (Von Der Linden et al. 2007). The new estimations of the flux of ZwCl 1665 still locate it slightly above the eeHIFLUGCS flux limit.

Our joint X-ray and optical analysis of the three components in observation RXC J0034.6-0208 indicates that two of such structures are located at similar redshift $(z \sim 0.05)$, while the third one is at a higher redshift $(z \sim 0.08)$. The X-ray peaks of the three components nicely coincide with giant elliptical galaxies located at different redshifts, and the galaxy redshift distribution is bi-modal. However, XMM-Newton presented some anomalies while observing this target: there is no data for the MOS1 camera and the X-ray spectral results of two of the three components differ between the MOS2 and pn cameras. The poor quality of the X-ray observation does not allow us to perform a deeper and joint analysis of this system with the available Planck data, as in Planck Collaboration VI (2013). A deeper XMM-Newton observation will provide us with a better understanding of this system: with the iron $\mathrm{K}$ lines, the redshift of the components will be constrained, and cluster residual emission will be modelled with higher precision. This will allow us to understand if the different components are part of the same supercluster structure or not.

The global properties of the galaxy groups studied in this work are in good agreement with measurements for a sample of groups at lower redshift ranges and with scaling relations fitted to those samples. The main differences between our derived fluxes and luminosities, either from ROSAT or XMM-Newton, may be explained by the distinct techniques and data used.

We have found that more than $1 \%$ of $~ 240$ eeHIFLUGCS clusters are the result of projection effects. This percentage represents a firm lower limit to the fraction of contaminated clusters. Further work is needed in order to assess the contamination fraction of the full eeHIFLUGCS sample. This will help to determine the level of contamination of low-resolution catalogues, like those that will be available from eROSITA.

In summary, our results show that low-angular-resolution X-ray surveys can produce biased cluster catalogues due to cluster-projection effects. This bias is three-fold: fluxes are biased high, cluster numbers are biased low, and redshifts are biased high or low. In this work, we show that with X-ray follow up using higher-spatial-resolution instruments we can iden- tify multiple cluster components. We also demonstrate that given sufficient spectral resolution and collected X-ray photons, X-ray redshifts can robustly separate projected components. In general, X-ray spectral analysis represents an independent means to determine the redshift also for less peculiar clusters in lowangular-resolution X-ray cluster catalogues. This implies that with XMM-Newton, Chandra, and XRISM follow-up of subsamples of clusters to be discovered with eROSITA, we will be able to quantify and correct for those biases.

Acknowledgements. The authors would like to thank the anonymous referee for the feedback on the manuscript, as well as Jens Erler for his assistance. MERC, FP, and THR acknowledge support from the German Aerospace Agency (DLR) with funds from the Ministry of Economy and Technology (BMWi) through grant 50 OR 1514. KM is supported by the International Max Planck Research School (IMPRS) for Astronomy and Astrophysics at the Universities of Bonn and Cologne and the Bonn-Cologne Graduate School (BCGS) of Physics and Astronomy. L.L. acknowledges support from the Chandra X-ray Center through NASA contract NNX17AD83G. GS acknowledges support by the National Aeronautics and Space Administration (NASA) through Chandra Award Number GO4-15129X issued by the Chandra X-ray Observatory Center (CXC), which is operated by the Smithsonian Astrophysical Observatory (SAO) for and on behalf of NASA under contract NAS8-03060. Preliminary work on one of the targets was done by University of Bonn student Vyoma Muralidhara. This research has made use of: the NASA/IPAC Extragalactic Database (NED) which is operated by the Jet Propulsion Laboratory, California Institute of Technology, under contract with the National Aeronautics and Space Administration; and the VizieR catalogue access tool, CDS, Strasbourg, France.

\section{References}

Abell, G. O. 1958, ApJS, 3, 211

Asplund, M., Grevesse, N., Sauval, A. J., \& Scott, P. 2009, ARA\&A, 47, 481 Banerjee, P., Szabo, T., Pierpaoli, E., et al. 2018, Nature, 58, 61

Bertin, E., \& Arnouts, S. 1996, A\&AS, 117, 393

Böhringer, H., \& Werner, N. 2010, A\&ARv, 18, 127

Böhringer, H., Voges, W., Huchra, J. P., et al. 2000, ApJS, 129, 435

Böhringer, H., Schuecker, P., Guzzo, L., et al. 2001, A\&A, 369, 826 Böhringer, H., Schuecker, P., Guzzo, L., et al. 2004, A\&A, 425, 367 Böhringer, H., Chon, G., Collins, C. A., et al. 2013, A\&A, 555, A30 Boller, T., Freyberg, M. J., Trümper, J., et al. 2016, A\&A, 588, A103 Borm, K., Reiprich, T. H., Mohammed, I., \& Lovisari, L. 2014, A\&A, 567, A65 Carter, J. A., \& Sembay, S. 2008, A\&A, 489, 837

Carter, J. A., Sembay, S., \& Read, A. M. 2011, A\&A, 527, A115 Chambers, K. C., \& Pan-STARRS Team 2016, Am. Astron. Soc. Meet. Abstr., 227, 324.07

Chon, G., Böhringer, H., \& Nowak, N. 2013, MNRAS, 429, 3272

Clerc, N., Merloni, A., Zhang, Y.-Y., et al. 2016, MNRAS, 463, 4490

Clerc, N., Ramos-Ceja, M. E., Ridl, J., et al. 2018, A\&A, 617, A92

Costanzi, M., Rozo, E., Rykoff, E. S., et al. 2019, MNRAS, 482, 490

Cruddace, R., Voges, W., Böhringer, H., et al. 2002, ApJS, 140, 239

Dark Energy Survey Collaboration (Abbott, T., et al.) 2016, MNRAS, 460, 1270 de Jong, R. S., Barden, S., Bellido-Tirado, O., et al. 2014, in Ground-based and Airborne Instrumentation for Astronomy V, Proc. SPIE, 9147, 91470M

De Luca, A., \& Molendi, S. 2004, A\&A, 419, 837

Ebeling, H., Edge, A. C., Bohringer, H., et al. 1998, MNRAS, 301, 881

Giodini, S., Lovisari, L., Pointecouteau, E., et al. 2013, Space Sci. Rev., 177, 247 Kuntz, K. D., \& Snowden, S. L. 2008, A\&A, 478, 575

Leccardi, A., \& Molendi, S. 2008, A\&A, 486, 359

Liu, A., Yu, H., Diaferio, A., et al. 2018, ApJ, 863, 102

Lloyd-Davies, E. J., Romer, A. K., Mehrtens, N., et al. 2011, MNRAS, 418, 14

Lovisari, L., \& Reiprich, T. H. 2019, MNRAS, 483, 540

Lovisari, L., Reiprich, T. H., \& Schellenberger, G. 2015, A\&A, 573, A118

McCammon, D., Almy, R., Apodaca, E., et al. 2002, ApJ, 576, 188

Merloni, A., Predehl, P., Becker, W., et al. 2012, ArXiv e-prints [arXiv:1209.3114]

Ochsenbein, F., Bauer, P., \& Marcout, J. 2000, A\&AS, 143, 23

Pacaud, F., Pierre, M., Refregier, A., et al. 2006, MNRAS, 372, 578

Peterson, J. R., \& Fabian, A. C. 2006, Phys. Rep., 427, 1

Piffaretti, R., Arnaud, M., Pratt, G. W., Pointecouteau, E., \& Melin, J.-B. 2011, A\&A, 534, A109

Pillepich, A., Porciani, C., \& Reiprich, T. H. 2012, MNRAS, 422, 44

Pillepich, A., Reiprich, T. H., Porciani, C., Borm, K., \& Merloni, A. 2018, MNRAS, 481, 613

Planck Collaboration VI. 2013, A\&A, 550, A132 
Planck Collaboration XXVII. 2016, A\&A, 594, A27

Pratt, G. W., \& Arnaud, M. 2002, A\&A, 394, 375

Predehl, P., Andritschke, R., Böhringer, H., et al. 2010, in Space Telescopes and Instrumentation 2010: Ultraviolet to Gamma Ray, Proc. SPIE, 7732, 77320U Reiprich, T. H. 2017, Astron. Nachr., 338, 349

Reiprich, T. H., Sarazin, C. L., Kempner, J. C., \& Tittley, E. 2004, ApJ, 608, 179 Reichert, A., Böhringer, H., Fassbender, R., \& Mühlegger, M. 2011, A\&A, 535, A4

Sarazin, C. L. 1986, Rev. Mod. Phys., 58, 1

Schellenberger, G., \& Reiprich, T. H. 2017, MNRAS, 469, 3738

Shanks, T., Metcalfe, N., Chehade, B., et al. 2015, MNRAS, 451, 4238

Shectman, S. A. 1985, ApJS, 57, 77

Smith, R. K., Brickhouse, N. S., Liedahl, D. A., \& Raymond, J. C. 2001, ApJ, 556, L91

Snowden, S. L., Mushotzky, R. F., Kuntz, K. D., \& Davis, D. S. 2008, A\&A, 478,615

Starck, J. L., Murtagh, F. D., \& Bijaoui, A. 1998, Image Processing and Data Analysis (Cambridge University Press)

Struble, M. F., \& Rood, H. J. 1999, ApJS, 125, 35

Tashiro, M., Maejima, H., Toda, K., et al. 2018, in Space Telescopes and Instrumentation 2018: Ultraviolet to Gamma Ray, SPIE Conf. Ser., 10699 1069922

Truemper, J. 1992, QJRAS, 33, 165

Truemper, J. 1993, Science, 260, 1769

Voges, W., Aschenbach, B., Boller, T., et al. 1999, A\&A, 349, 389

Voges, W., Aschenbach, B., Boller, T., et al. 2000, VizieR Online Data Catalog: IX/029

Von Der Linden, A., Best, P. N., Kauffmann, G., \& White, S. D. M. 2007, MNRAS, 379, 867

Wen, Z. L., Han, J. L., \& Liu, F. S. 2012, ApJS, 199, 34

Willingale, R., Starling, R. L. C., Beardmore, A. P., Tanvir, N. R., \& O'Brien, P. T. 2013, MNRAS, 431, 394

Xu, W., Ramos-Ceja, M. E., Pacaud, F., Reiprich, T. H., \& Erben, T. 2018, A\&A, 619, A162

Yu, H., Tozzi, P., Borgani, S., Rosati, P., \& Zhu, Z.-H. 2011, A\&A, 529, A65

Zhang, Y.-Y., Böhringer, H., Finoguenov, A., et al. 2006, A\&A, 456, 55

Zhang, Y.-Y., Reiprich, T. H., Finoguenov, A., Hudson, D. S., \& Sarazin, C. L. 2009, ApJ, 699, 1178

Zhang, Y.-Y., Reiprich, T. H., Schneider, P., et al. 2017, A\&A, 599, A138

\section{Appendix A: Detailed analysis of RXC J0034.6-0208}

Table A.1. Redshift estimations from the X-ray spectral analysis for the components of RXC J0034.6-0208.

\begin{tabular}{lcc}
\hline \hline Components & MOS2 & pn \\
\hline SW & $0.086_{-0.015}^{+0.007}$ & $0.081_{-0.012}^{+0.012}$ \\
E & - & $0.055_{-0.020}^{+0.020}$ \\
NW & $0.064_{-0.008}^{+0.005}$ & $0.026_{-0.010}^{+0.006}$ \\
\hline
\end{tabular}

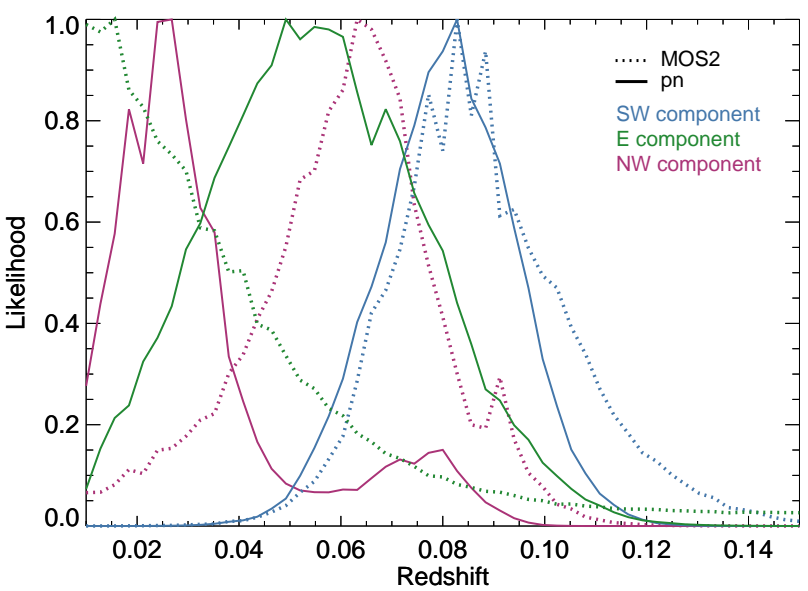

Fig. A.1. Variation of $\mathcal{L}$ as a function of the redshift for the different extended components in the RXC J0034.6-0208 observation. Solid and dotted lines represent the MOS2 and pn detectors, respectively; the blue, green, and purple lines show the results for the southwestern, eastern, and northwestern components, respectively.

For the analysis of the RXC J0034.6-0208 cluster system we use the extracted spectra in circles of 3 arcmin radius centred on each cluster component. The quality of the data does not allow us to detect the iron K or L complex in either of the structures. We fitted the spectra for each detector separately as explained in Sect. 3.2. We report the results for the redshift analysis in Table A.1.

The redshift measurements for each detector in the southwestern component are consistent within $1 \sigma$. A joint fitting of all available instruments gives a consistent redshift result $(z=$ 0.082, see Sect. 4.2). For the northwestern structure, the result of redshift estimation with MOS2 is higher than the results with pn, although they are consistent at the $3 \sigma$ level. In this case, a joint fit gives $z=0.028$, indicating that the pn spectrum determines the redshift estimate, which is not surprising given the much higher sensitivity of pn. In a similar way, the redshift results of pn of the eastern component settle the final value of the joint fitting $(z=0.045)$ since the values of MOS2 cannot be constrained. Figure A. 1 shows the variation of $\mathcal{L}$ for the individual fits of the three components.

The redshift discrepancies between the detectors suggest that there is a systematic problem in the observation. This is supported by the lack of MOS1 data in the observation. 